\title{
Splitting of the Einstein Field Equations with Respect to the $(1+1+3)$ Threading of a $5 D$ Universe
}

\author{
Aurel Bejancu and Hani Reda Farran*
}

(Dedicated to the memory of Prof. Dr. Aurel BEJANCU (1946 - 2020))

\begin{abstract}
We obtain a new and simple splitting of Einstein field equations with respect to the $(1+1+3)$ threading of a $5 D$ universe $(\bar{M}, \bar{g})$. The study is based on the spatial tensor fields and on the Riemannian spatial connection, which behave as $3 D$ geometric objects. All the equations are expressed with respect to the adapted frame field and the adapted coframe field induced by the $(1+1+3)$ threading of $(\bar{M}, \bar{g})$. In particular, we obtain the splitting of the Einstein field equations in a $5 D$ Robertson-Walker universe.
\end{abstract}

Keywords: Einstein field equations, spatial tensor fields, 3D geometric objects, $5 D$ Robertson-Walker universe.

AMS Subject Classification (2020): Primary: $53 Z 05$.

\section{Introduction}

This paper is a continuation of the papers $[1,2]$ of the first author devoted to Raychaudhuri equations and to equations of motion in a $5 D$ universe. According to the new approach presented in the above mentioned papers, the $5 D$ universe $\bar{M}=M \times K$ is studied by means of the submersion of $\bar{M}$ on the $4 D$ spacetime $M$. We should stress that in all the other theories of a $5 D$ universe, the study was performed by considering $M$ as a submanifold embedded in $\bar{M}$ ( cf. [4, 5])

The spatial tensor fields, the kinematic quantities and the covariant derivatives induced by the Riemannian spatial connection, are the main tools used in the paper. We start from the coordinate-free form of the Einstein field equations (EFE) and by using the adapted frame field on $\bar{M}$, we obtain the local form of the 15 (EFE). As an application we write down the (EFE) for a $5 D$ Robertson-Walker universe.

Now, we outline the content of the paper. In Sect. 2 we recall from [1] the geometric configuration of a $5 D$ universe. The structure equations induced by the $(1+1+3)$ threading of a $5 D$ universe are obtained in Sect.3 (cf. (3.9)-(3.12)). These equations are splitting in Gauss equations, Codazzi equations and Ricci equations for the spatial distribution of the $5 D$ universe $(\bar{M}, \bar{g})$. In Sect. 4 we obtain the local components of the Ricci tensor of $(\bar{M}, \bar{g})$ with respect to the adapted frame field (cf.(4.6)), and the scalar curvature of $(\bar{M}, \bar{g})$ (cf. (4.9)). The spatial Ricci tensor and the spatial scalar curvature characterize the geometry of the spatial distribution, which in our study is not necessarily integrable. These geometric objects enable us in Sect.5 to proceed to the splitting of (EFE) in $(\bar{M}, \bar{g})$ (cf. (5.6), (5.8)-(5.12)). We succeed to pick out the six spatial Einstein field equations (SEFE) (5.6) in which appears for the first time in literature the spatial Einstein gravitational tensor field. In Sect.6 we apply the general theory to the $5 D$ Robertson-Walker universe. We should note that equations (6.13), (6.16) and (6.17) show us that some local components of the energy-momentum tensor must vanish, and the warping function should be a solution for two PDE. We close the paper with Conclusions and an Appendix. 


\section{The geometric configuration of a $5 D$ Universe}

In this section we present the main geometric objects induced by the $(1+1+3)$ threading of a $5 D$ universe $[1,2]$.

Let $M$ and $K$ be manifolds of dimension four and one respectively, and $\bar{M}=M \times K$ be the product bundle over $M$ with fibre $K$. We have a coordinate system $\left(x^{a}\right)=\left(x^{i}, x^{4}\right)$ on $\bar{M}$, where $x^{4}$ is the fibre coordinate and $\left(x^{i}\right)$ is a coordinate system on $M$. Suppose that $\bar{M}$ is endowed with a Lorentz metric $\bar{g}$ such that

$$
\bar{g}(\eta, \eta)=\Psi^{2},
$$

where $\eta$ is a globally defined vector field on $\bar{M}$ that is locally given by $\partial / \partial x^{4}$. Denote by $\mathcal{V} \bar{M}$ the line bundle spanned by $\eta$ and by $\mathcal{H} \bar{M}$ the complementary orthogonal vector bundle to $\mathcal{V} \bar{M}$ in the tangent bundle $T \bar{M}$ of $\bar{M}$. We call $\mathcal{V} \bar{M}$ (resp. $\mathcal{H} \bar{M}$ ) the vertical distribution (resp.horizontal distribution) on $\bar{M}$. Now, suppose that on $M$ exists a globally defined horizontal vector field $U$ such that locally we have $U=\partial / \partial x^{0}$. Then it is proved in [1] that there exists a globally defined horizontal vector field $\xi$ on $\bar{M}$, which is locally given by

$$
\frac{\delta}{\delta x^{0}}=\frac{\partial}{\partial x^{0}}-A_{0} \frac{\partial}{\partial x^{4}}
$$

and it is timelike with respect to $\bar{g}$, that is, we have

$$
\bar{g}\left(\frac{\delta}{\delta x^{0}}, \frac{\delta}{\delta x^{0}}\right)=-\Phi^{2} .
$$

The timelike vector field $\xi$ and the spacelike vector field $\eta$ are called the $4 D$ velocity and the $5 D$ velocity, respectively. The line bundle spanned by $\xi$ on $\bar{M}$ is denoted by $\mathcal{T} \bar{M}$ and it is called temporal distribution. The complementary orthogonal bundle to $\mathcal{T} \bar{M}$ in $\mathcal{H} \bar{M}$ is denoted by $\mathcal{S} \bar{M}$, and it is called the spatial distribution. Then we have the orthogonal decomposition of the tangent bundle of $\bar{M}$

$$
T \bar{M}=\mathcal{T} \bar{M} \oplus \mathcal{S} \bar{M} \oplus \mathcal{V} \bar{M} .
$$

Throughout the paper we use the following ranges of indices: $a, b, c, \ldots \in\{0,1,2,3,4\}, i, j, k, \ldots \in\{0,1,2,3\}$, and $\alpha, \beta, \gamma, \ldots \in\{1,2,3\}$. For any vector bundle $E$ over $\bar{M}$ denote by $\Gamma(E)$ the $\mathcal{F}(\bar{M})$-module of smooth sections of $E$, where $\mathcal{F}(\bar{M})$ is the algebra of smooth functions on $\bar{M}$.Also, to express in a simple way some long formulas, we use notations like this:

$$
\mathcal{A}_{(\beta \gamma)}\left\{\omega_{\alpha \beta} \Phi_{\gamma}+\omega_{\alpha \beta \mid \gamma}\right\}=\omega_{\alpha \beta} \Phi_{\gamma}-\omega_{\alpha \gamma} \Phi_{\beta}+\omega_{\alpha \beta \mid \gamma}-\omega_{\alpha \gamma \mid \beta} .
$$

The decomposition (2.4) enables us to use in the study the adapted frame field $\left\{\delta / \delta x^{0}, \delta / \delta x^{\alpha}, \partial / \partial x^{4}\right\}$ and the adapted coframe field $\left\{\delta x^{0}, d x^{\alpha}, \delta x^{4}\right\}$ on $\bar{M}$, where we put

$$
\begin{aligned}
& \text { (a) } \frac{\delta}{\delta x^{\alpha}}=\frac{\partial}{\partial x^{\alpha}}-B_{\alpha} \frac{\delta}{\delta x^{0}}-A_{\alpha} \frac{\partial}{\partial x^{4}}, \\
& \text { (b) } \delta x^{0}=d x^{0}+B_{\alpha} d x^{\alpha}, \quad \text { (c) } \delta x^{4}=d x^{4}+A_{i} d x^{i} .
\end{aligned}
$$

As the fibres of $\mathcal{T} \bar{M}$ are timelike, the Lorentz metric $\bar{g}$ induces on $\mathcal{S}(\bar{M})$ a Riemannian metric $h$ with local components

$$
h_{\alpha \beta}=h\left(\frac{\delta}{\delta x^{\beta}}, \frac{\delta}{\delta x^{\alpha}}\right)=\bar{g}\left(\frac{\delta}{\delta x^{\beta}}, \frac{\delta}{\delta x^{\alpha}}\right) .
$$

Thus, the line element on $\bar{M}$ with respect to the adapted coframe field has the form

$$
d \bar{s}^{2}=-\Phi^{2}\left(\delta x^{0}\right)^{2}+h_{\alpha \beta} d x^{\alpha} d x^{\beta}+\Psi^{2}\left(\delta x^{4}\right)^{2} .
$$

Next, we recall from [1] the kinematic quantities on $\bar{M}$.

First, the $4 D$ vorticity $\omega_{\alpha \beta}$ and the $5 D$ vorticity $\eta_{\alpha \beta}$ are given by

$$
\begin{aligned}
& \text { (a) } \omega_{\alpha \beta}=\frac{1}{2} \mathcal{A}_{(\alpha \beta)}\left\{\frac{\delta B_{\beta}}{\delta x^{\alpha}}\right\}, \\
& \text { (b) } \eta_{\alpha \beta}=\frac{1}{2} \mathcal{A}_{(\alpha \beta)}\left\{\frac{\delta A_{\beta}}{\delta x^{\alpha}}+B_{\alpha} \frac{\delta A_{0}}{\delta x^{\beta}}\right\} .
\end{aligned}
$$

Then, the $4 D$ expansion tensor field $\Theta_{\alpha \beta}$ and the $5 D$ expansion tensor field $K_{\alpha \beta}$ are expressed as follows: 


$$
\text { (a) } \Theta_{\alpha \beta}=\frac{1}{2} \frac{\delta h_{\alpha \beta}}{\delta x^{0}}, \quad \text { (b) } K_{\alpha \beta}=\frac{1}{2} \frac{\partial h_{\alpha \beta}}{\partial x^{4}} \text {. }
$$

The trace of these spatial tensor fields define the $4 D$ expansion function $\Theta$ and the $5 D$ expansion function $K$ given by

$$
\text { (a) } \Theta=\Theta_{\alpha \beta} h^{\alpha \beta}, \quad \text { (b) } K=K_{\alpha \beta} h^{\alpha \beta} \text {. }
$$

Also, the trace-free spatial tensor fields $\sigma_{\alpha \beta}$ and $H_{\alpha \beta}$ given by

$$
\text { (a) } \sigma_{\alpha \beta}=\Theta_{\alpha \beta}-\frac{1}{3} \Theta h_{\alpha \beta}, \quad \text { (b) } H_{\alpha \beta}=K_{\alpha \beta}-\frac{1}{3} K h_{\alpha \beta}
$$

are called the $4 D$ shear tensor field and the $5 D$ shear tensor field on $\bar{M}$, respectively.

The Lie brackets of vector fields from the adapted frame field are expressed as follows:

$$
\begin{aligned}
& \text { (a) }\left[\frac{\delta}{\delta x^{\beta}}, \frac{\delta}{\delta x^{\alpha}}\right]=2 \omega_{\alpha \beta} \frac{\delta}{\delta x^{0}}+2 \eta_{\alpha \beta} \frac{\partial}{\partial x^{4}} \\
& \text { (b) }\left[\frac{\delta}{\delta x^{\alpha}}, \frac{\delta}{\delta x^{0}}\right]=b_{\alpha} \frac{\delta}{\delta x^{0}}+a_{\alpha} \frac{\partial}{\partial x^{4}}, \\
& \text { (c) }\left[\frac{\delta}{\delta x^{\alpha}}, \frac{\partial}{\partial x^{4}}\right]=d_{\alpha} \frac{\delta}{\delta x^{0}}+c_{\alpha} \frac{\partial}{\partial x^{4}}, \\
& \text { (d) }\left[\frac{\delta}{\delta x^{0}}, \frac{\delta}{\delta x^{4}}\right]=a_{0} \frac{\partial}{\partial x^{4}},
\end{aligned}
$$

From (2.12d) we see that the distribution $\mathcal{T} \bar{M} \oplus \mathcal{V} \bar{M}$ is integrable.

Next, we denote by $\bar{\nabla}$ the Levi-Civita connection defined by $\bar{g}$ on $\bar{M}$, and recall from [1] the Riemannian spatial connection $\nabla$ on the spatial distribution $\mathcal{S} \bar{M}$ given by

$$
\nabla_{X} \mathcal{S} Y=\mathcal{S} \bar{\nabla}_{X} \mathcal{S} Y, \quad \forall X, Y \in \Gamma(T \bar{M})
$$

where $\mathcal{S}$ is the projection morphism of $T \bar{M}$ on $\mathcal{S} \bar{M}$ with respect to the decomposition (2.4). Locally, $\nabla$ is given by

$$
\begin{gathered}
\text { (a) } \nabla_{\frac{\delta}{\delta x^{\beta}}} \frac{\delta}{\delta x^{\alpha}}=\Gamma_{\alpha \beta}^{\gamma} \frac{\delta}{\delta x^{\gamma}}, \quad(b) \quad \nabla_{\frac{\partial}{\partial x^{0}}} \frac{\delta}{\delta x^{\alpha}}=\Gamma_{\alpha}^{\gamma} \frac{\delta}{\delta x^{\gamma}} \\
\text { (c) } \nabla_{\frac{\partial}{\partial x^{4}} \frac{\delta}{\delta x^{\alpha}}}=\Gamma_{\alpha}^{\gamma} \frac{\delta}{\delta x^{\gamma}}
\end{gathered}
$$

where we put

$$
\begin{gathered}
\text { (a) } \Gamma_{\alpha \beta}^{\gamma}=\frac{1}{2} h^{\gamma \mu}\left\{\frac{\delta h_{\mu \alpha}}{\delta x^{\beta}}+\frac{\delta h_{\mu \beta}}{\delta x^{\alpha}}-\frac{\delta h_{\alpha \beta}}{\delta x^{\mu}}\right\}, \\
\text { (b) } \Gamma_{\alpha 0}^{\gamma}=\Theta_{\alpha}^{\gamma}+\Phi^{2} \omega_{\alpha}^{\gamma}, \quad \text { (c) } \Gamma_{\alpha 4}^{\gamma}=K_{\alpha}^{\gamma}-\Psi^{2} \eta_{\alpha}^{\gamma} .
\end{gathered}
$$

The pair $(\bar{M}, \bar{g})$ with the geometric configuration described in this section is called a $5 D$ universe. As it was explained in [1] this $5 D$ universe is different from the ones considered in both the brane-world theory $[3,4]$ and the space-time-matter theory $[5,6]$.

We close this section with the local expressions of Levi-Civita connection $\bar{\nabla}$ on the $5 D$ universe $(\bar{M}, \bar{g})$ (cf. [1]): 
(a) $\bar{\nabla}_{\frac{\delta}{\delta x^{\beta}}} \frac{\delta}{\delta x^{\alpha}}=\Gamma_{\alpha}^{\gamma} \frac{\delta}{\delta x^{\gamma}}+\left(\omega_{\alpha \beta}+\Phi^{-2} \Theta_{\alpha \beta}\right) \frac{\delta}{\delta x^{0}}$

$$
+\left(\eta_{\alpha \beta}-\Psi^{-2} K_{\alpha \beta}\right) \frac{\partial}{\partial x^{4}},
$$

(b) $\bar{\nabla}_{\frac{\delta}{\delta x^{0}}} \frac{\delta}{\delta x^{\alpha}}=\Gamma_{\alpha}^{\gamma} 0 \frac{\delta}{\delta x^{\gamma}}+\left(\Phi_{\alpha}-b_{\alpha}\right) \frac{\delta}{\delta x^{0}}$

$$
+\frac{1}{2}\left(\Phi^{2} d_{\alpha} \Psi^{-2}-a_{\alpha}\right) \frac{\partial}{\partial x^{4}},
$$

(c) $\bar{\nabla} \frac{\partial}{\partial x^{4}} \frac{\delta}{\delta x^{\alpha}}=\Gamma_{\alpha}^{\gamma} \frac{\delta}{\delta x^{\gamma}}+\frac{1}{2}\left(\Psi^{2} a_{\alpha} \Phi^{-2}-d_{\alpha}\right) \frac{\delta}{\delta x^{0}}$

$$
+\left(\Psi_{\alpha}-c_{\alpha}\right) \frac{\partial}{\partial x^{4}}
$$

(d) $\bar{\nabla} \frac{\delta}{\delta x^{\alpha}} \frac{\delta}{\delta x^{0}}=\Gamma_{\alpha}^{\gamma} \frac{\delta}{\delta x^{\gamma}}+\phi_{\alpha} \frac{\delta}{\delta x^{0}}+\frac{1}{2}\left(\Phi^{2} d_{\alpha} \Psi^{-2}+a_{\alpha}\right) \frac{\partial}{\partial x^{4}}$,

(e) $\bar{\nabla} \frac{\delta}{\delta x^{\alpha}} \frac{\partial}{\partial x^{4}}=\Gamma_{\alpha}^{\gamma} \frac{\delta}{\delta x^{\gamma}}+\frac{1}{2}\left(\Psi^{2} a_{\alpha} \Phi^{-2}+d_{\alpha}\right) \frac{\delta}{\delta x^{0}}+\Psi_{\alpha} \frac{\partial}{\partial x^{4}}$,

(f) $\bar{\nabla}_{\frac{\partial}{\partial x^{4}}} \frac{\delta}{\delta x^{0}}=\frac{1}{2}\left(\Psi^{2} a^{\gamma}-\Phi^{2} d^{\gamma}\right) \frac{\delta}{\delta x^{\gamma}}+\phi_{4} \frac{\delta}{\delta x^{0}}+\left(\Psi_{0}-a_{0}\right) \frac{\partial}{\partial x^{4}}$,

(g) $\bar{\nabla}_{\frac{\delta}{\delta x^{0}}} \frac{\partial}{\partial x^{4}}=\frac{1}{2}\left(\Psi^{2} a^{\gamma}-\Phi^{2} d^{\gamma}\right) \frac{\delta}{\delta x^{\gamma}}+\phi_{4} \frac{\delta}{\delta x^{0}}+\Psi_{0} \frac{\partial}{\partial x^{4}}$,

(h) $\bar{\nabla}_{\frac{\delta}{\delta x^{0}}} \frac{\delta}{\delta x^{0}}=\Phi^{2}\left(\phi^{\gamma}-b^{\gamma}\right) \frac{\delta}{\delta x^{\gamma}}+\phi_{0} \frac{\delta}{\delta x^{0}}+\Phi^{2} \Phi_{4} \Psi^{-2} \frac{\partial}{\partial x^{4}}$,

(i) $\bar{\nabla} \frac{\partial}{\partial x^{4}} \frac{\partial}{\partial x^{4}}=\Psi^{2}\left(c^{\gamma}-\Psi^{\gamma}\right) \frac{\delta}{\delta x^{\gamma}}+\Psi^{2}\left(\psi_{0}-a_{0}\right) \Phi^{-2} \frac{\delta}{\delta x^{0}}+\Psi_{4} \frac{\partial}{\partial x^{4}}$,

where we put

$$
\begin{array}{lll}
\text { (a) } \Phi_{i}=\Phi^{-1} \frac{\delta \Phi}{\delta x^{i}}, & \text { (b) } \Psi_{i}=\Psi^{-1} \frac{\delta \Psi}{\delta x^{i}} \\
\text { (c) } \Phi_{4}=\Phi^{-1} \frac{\partial \Phi}{\partial x^{4}}, & \text { (d) } \Psi_{4}=\Psi^{-1} \frac{\partial \Psi}{\partial x^{4}} .
\end{array}
$$

\section{Structure equations induced by the $(1+1+3)$ threading of a $5 D$ Universe}

The Riemannian spatial connection $\nabla$ defined in [1] is a metric linear connection on the spatial distribution $\mathcal{S} \bar{M}$, and its curvature tensor field is given by

$$
R(X, Y, \mathcal{S} Z)=\nabla_{X} \nabla_{Y} \mathcal{S} Z-\nabla_{Y} \nabla_{X} \mathcal{S} Z-\nabla_{[X, Y]} \mathcal{S} Z,
$$

for all $X, Y, Z \in \Gamma(T \bar{M})$. Locally, we put:

$$
\begin{aligned}
& R\left(\frac{\delta}{\delta x^{\gamma}}, \frac{\delta}{\delta x^{\beta}}, \frac{\delta}{\delta x^{\alpha}}\right)=R_{\alpha \beta \gamma}^{\mu} \frac{\delta}{\delta x^{\mu}}, \\
& R\left(\frac{\delta}{\delta x^{\gamma}}, \frac{\delta}{\delta x^{0}}, \frac{\delta}{\delta x^{\alpha}}\right)=R_{\alpha 0 \gamma}^{\mu} \frac{\delta}{\delta x^{\mu}}, \\
& R\left(\frac{\delta}{\delta x^{\gamma}}, \frac{\partial}{\partial x^{4}}, \frac{\delta}{\delta x^{\alpha}}\right)=R_{\alpha 4 \gamma}^{\mu} \frac{\delta}{\delta x^{\mu}}, \\
& R\left(\frac{\delta}{\delta x^{0}}, \frac{\partial}{\partial x^{4}}, \frac{\delta}{\delta x^{\alpha}}\right)=R_{\alpha 40}^{\mu} \frac{\delta}{\delta x^{\mu}} .
\end{aligned}
$$

Then by direct calculations using (3.1), (3.2), (2.12) and (2.14) we obtain

$$
\begin{array}{r}
\text { (a) } R_{\alpha \beta \gamma}^{\mu}=\frac{\delta \Gamma_{\alpha \beta}^{\mu}}{\delta x^{\gamma}}-\frac{\delta \Gamma_{\alpha \gamma}^{\mu}}{\delta x^{\beta}}+\Gamma_{\alpha \beta}^{\nu} \Gamma_{\nu \gamma}^{\mu}-\Gamma_{\alpha \gamma}^{\nu} \Gamma_{\nu \beta}^{\mu} \\
-2 \omega_{\beta \gamma} \Gamma_{\alpha 0}^{\mu}-2 \eta_{\beta \gamma} \Gamma_{\alpha 4}^{\mu}, \\
\text { (b) } R_{\alpha 0 \gamma}^{\mu}=\frac{\delta \Gamma_{\alpha 0}^{\mu}}{\delta x^{\gamma}}-\frac{\delta \Gamma_{\alpha \gamma}^{\mu}}{\delta x^{0}}+\Gamma_{\alpha{ }_{0}}^{\nu} \Gamma_{\nu \gamma}^{\mu}-\Gamma_{\alpha \gamma}^{\nu} \Gamma_{\nu 0}^{\mu} \\
-b_{\gamma} \Gamma_{\alpha 0}^{\mu}-a_{\gamma} \Gamma_{\alpha 4}^{\mu}, \\
\text { (c) } R_{\alpha 4 \gamma}^{\mu}=\frac{\delta \Gamma_{\alpha 4}^{\mu}-\frac{\partial \Gamma_{\alpha \gamma}^{\mu}}{\delta x^{4}}+\Gamma_{\alpha 4}^{\nu} \Gamma_{\nu \gamma}^{\mu}-\Gamma_{\alpha \gamma}^{\nu} \Gamma_{\nu 4}^{\mu}}{-d_{\gamma} \Gamma_{\alpha 0}^{\mu}-c_{\gamma} \Gamma_{\alpha 4}^{\mu},} \\
\text { (d) } R_{\alpha 40}^{\mu}=\frac{\delta \Gamma_{\alpha 4}^{\mu}}{\delta x^{0}}-\frac{\partial \Gamma_{\alpha 0}^{\mu}+\Gamma_{\alpha 4}^{\nu} \Gamma_{\nu 0}^{\mu}}{\partial x^{4}}+\Gamma_{\alpha 0}^{\nu} \Gamma_{\nu 4}^{\mu}-a_{0} \Gamma_{\alpha 4}^{\mu} . \\
-
\end{array}
$$


Now, let $\bar{R}$ be the curvature tensor field of the Levi-Civia connection $\bar{\nabla}$ on the $5 D$ universe $(\bar{M}, \bar{g})$. Then with respect to the adapted frame field $\left\{\frac{\delta}{\delta x^{0}}, \frac{\delta}{\delta x^{\alpha}}, \frac{\partial}{\partial x^{4}}\right\}$ we express $\bar{R}$ as follows:

(a) $\bar{R}\left(\frac{\delta}{\delta x^{\gamma}}, \frac{\delta}{\delta x^{\beta}}, \frac{\delta}{\delta x^{\alpha}}\right)=\bar{R}_{\alpha \beta \gamma}^{0} \frac{\delta}{\delta x^{0}}+\bar{R}_{\alpha \beta \gamma}^{\mu} \frac{\delta}{\delta x^{\mu}}+\bar{R}_{\alpha \beta \gamma}^{4} \frac{\partial}{\partial x^{4}}$,

(b) $\bar{R}\left(\frac{\delta}{\delta x^{\gamma}}, \frac{\delta}{\delta x^{0}}, \frac{\delta}{\delta x^{\alpha}}\right)=\bar{R}_{\alpha 0 \gamma}^{0} \frac{\delta}{\delta x^{0}}+\bar{R}_{\alpha 0 \gamma}^{\mu} \frac{\delta}{\delta x^{\mu}}+\bar{R}_{\alpha 0 \gamma}^{4} \frac{\partial}{\partial x^{4}}$,

(c) $\bar{R}\left(\frac{\delta}{\delta x^{\gamma}}, \frac{\partial}{\partial x^{4}}, \frac{\delta}{\delta x^{\alpha}}\right)=\bar{R}_{\alpha 4 \gamma}^{0} \frac{\delta}{\delta x^{0}}+\bar{R}_{\alpha 4 \gamma}^{\mu} \frac{\delta}{\delta x^{\mu}}+\bar{R}_{\alpha 4 \gamma}^{4} \frac{\partial}{\partial x^{4}}$,

(d) $\bar{R}\left(\frac{\delta}{\delta x^{0}}, \frac{\partial}{\partial x^{4}}, \frac{\delta}{\delta x^{\alpha}}\right)=\bar{R}_{\alpha 40}^{0} \frac{\delta}{\delta x^{0}}+\bar{R}_{\alpha 40}^{\mu} \frac{\delta}{\delta x^{\mu}}+\bar{R}_{\alpha 40}^{4} \frac{\partial}{\partial x^{4}}$.

In this section we use the calculation performed in the Appendix. First, by comparing (3.4a) with $\left(A_{4}\right)$, we deduce that

$$
\begin{array}{r}
(a) \bar{R}_{\alpha \beta \gamma}^{\mu}=R_{\alpha \beta \gamma}^{\mu}+\mathcal{A}_{(\beta \gamma)}\left\{\left(\omega_{\alpha \beta}+\Phi^{-2} \theta_{\alpha \beta}\right) \Gamma_{\gamma 0}^{\mu}\right. \\
\left.+\left(\eta_{\alpha \beta}-\Psi^{-2} K_{\alpha \beta}\right) \Gamma_{\gamma 4}^{\mu}\right\} \\
(b) \bar{R}_{\alpha \beta \gamma}^{0}=\mathcal{A}_{(\beta \gamma)}\left\{\omega_{\alpha \beta \mid \gamma}+\Phi^{-2}\left(\theta_{\alpha \beta \mid \gamma}+\theta_{\alpha \gamma} \Phi_{\beta}\right)+\omega_{\alpha \beta} \Phi_{\gamma}\right. \\
\left.+\frac{1}{2}\left(\Psi^{2} a_{\gamma} \Phi^{-2}+d_{\gamma}\right)\left(\eta_{\alpha \beta}-\Psi^{-2} K_{\alpha \beta}\right)\right\} \\
-2\left(\Phi_{\alpha}-b_{\alpha}\right) \omega_{\beta \gamma}-\left(\Psi^{2} a_{\alpha} \Phi^{-2}-d_{\alpha}\right) \eta_{\beta \gamma}, \\
\text { (c) } \bar{R}_{\alpha \beta \gamma}^{4}=\mathcal{A}_{(\beta \gamma)}\left\{\eta_{\alpha \beta \mid \gamma}+\Psi^{-2}\left(K_{\alpha \gamma \mid \beta}+K_{\alpha \beta} \Psi_{\gamma}\right)+\eta_{\alpha \beta} \Psi_{\gamma}\right. \\
\left.+\frac{1}{2}\left(\Phi^{2} d_{\gamma} \Psi^{-2}+a_{\gamma}\right)\left(\omega_{\alpha \beta}+\Phi^{-2} \theta_{\alpha \beta}\right)\right\}-2\left(\psi_{\alpha}-c_{\alpha}\right) \eta_{\beta \gamma} \\
-\left(\Phi^{2} d_{\alpha} \Psi^{-2}-a_{\alpha}\right) \omega_{\beta \gamma} .
\end{array}
$$

Similarly, by comparing (3.4b), (3.4c) and (3.4d) with $\left(A_{5}\right),\left(A_{6}\right)$ and $\left(A_{7}\right)$ respectively, we obtain

$$
\begin{array}{r}
(a) \bar{R}_{\alpha 0 \gamma}^{\mu}=R_{\alpha 0 \gamma}^{\mu}+\left(\Phi_{\alpha}-b_{\alpha}\right) \Gamma_{\gamma 0}^{\mu} \\
+\frac{1}{2}\left(\Phi^{2} d_{\alpha} \Psi^{-2}-a_{\alpha}\right) \Gamma_{\gamma 4}^{\mu}-\left(\theta_{\alpha \gamma}+\Phi^{2} \omega_{\alpha \gamma}\right)\left(\Phi^{\mu}-b^{\mu}\right) \\
-\frac{1}{2}\left(\eta_{\alpha \gamma}-\Psi^{-2} K_{\alpha \gamma}\right)\left(\Psi^{2} a^{\mu}-\Phi^{2} d^{\mu}\right), \\
(b) \bar{R}_{\alpha 0 \gamma}^{0}=\Phi_{\alpha \mid \gamma}-b_{\alpha \mid \gamma}-\left(\omega_{\alpha \gamma}+\Phi^{-2} \theta_{\alpha \gamma}\right)_{\mid 0} \\
-\left(\omega_{\alpha \mu}+\Phi^{-2} \theta_{\alpha \mu}\right) \Gamma_{\gamma 0}^{\mu}+\left(\Phi_{\alpha}-b_{\alpha}\right)\left(\Phi_{\gamma}-b_{\gamma}\right) \\
-\Phi_{0}\left(\omega_{\alpha \gamma}+\Phi^{-2} \theta_{\alpha \gamma}\right)-\Phi_{4}\left(\eta_{\alpha \gamma}-\Psi^{-2} K_{\alpha \gamma}\right) \\
+\frac{1}{4}\left(3 d_{\alpha} a_{\gamma}-a_{\alpha} d_{\gamma}+\Phi^{2} d_{\alpha} d_{\gamma} \Psi^{-2}-3 \Psi^{2} a_{\alpha} a_{\gamma} \Phi^{-2}\right), \\
(c) \bar{R}_{\alpha}^{4} 0 \gamma=\frac{1}{2}\left(\Phi^{2} d_{\alpha} \Psi^{-2}-a_{\alpha}\right)_{\mid \gamma}-\left(\eta_{\alpha \gamma}-\Psi^{-2} K_{\alpha \gamma}\right){ }_{\mid 0} \\
-\left(\eta_{\alpha \mu}-\Psi^{-2} K_{\alpha \mu}\right) \Gamma_{\gamma 0}^{\mu}+\frac{1}{2}\left(\Phi_{\alpha}-b_{\alpha}\right)\left(\Phi^{2} d_{\gamma} \Psi^{-2}+a_{\gamma}\right) \\
+\frac{1}{2}\left(\Psi_{\gamma}-c_{\gamma}\right)\left(\Phi^{2} d_{\alpha} \Psi^{-2}-a_{\alpha}\right) \\
-\Phi^{2} \Phi_{4} \Psi^{-2}\left(\omega_{\alpha \gamma}+\Phi^{-2} \theta_{\alpha \gamma}\right)-\Psi_{0}\left(\eta_{\alpha \gamma}-\Psi^{-2} K_{\alpha \gamma}\right) \\
-\left(\Psi_{\alpha}-c_{\alpha}\right) a_{\gamma},
\end{array}
$$


(a) $\bar{R}_{\alpha 4 \gamma}^{\mu}=R_{\alpha 4 \gamma}^{\mu}+\frac{1}{2}\left(\Psi^{2} a_{\alpha} \Phi^{-2}-d_{\alpha}\right) \Gamma_{\gamma 0}^{\mu}+\left(\Psi_{\alpha}-c_{\alpha}\right) \Gamma_{\gamma 4}^{\mu}$

$$
\begin{gathered}
-\frac{1}{2}\left(\omega_{\alpha \gamma}+\Phi^{-2} \Theta_{\alpha \gamma}\right)\left(\Psi^{2} a^{\mu}-\Phi^{2} d^{\mu}\right) \\
+\left(\Psi^{2} \eta_{\alpha \gamma}-K_{\alpha \gamma}\right)\left(c^{\mu}-\Psi^{\mu}\right), \\
(b) \bar{R}_{\alpha{ }_{4 \gamma}}^{0}=\frac{1}{2}\left(\Psi^{2} a_{\alpha} \Phi^{-2}-d_{\alpha}\right)_{\mid \gamma}-\left(\omega_{\alpha \gamma}+\Phi^{2} \Theta_{\alpha \gamma}\right)_{\mid 4} \\
-\left(\omega_{\alpha \mu}+\Phi^{-2} \Theta_{\alpha \mu}\right) \Gamma_{\gamma 4}^{\mu}+\frac{1}{2}\left(\Psi^{2} a_{\alpha} \Phi^{-2}-d_{\alpha}\right) \Psi_{\gamma} \\
+\frac{1}{2}\left(\Psi_{\alpha}-c_{\alpha}\right)\left(\Psi^{2} a_{\alpha} \Phi^{-2}+d_{\alpha}\right)-\Phi_{4}\left(\omega_{\alpha \gamma}+\Phi^{-2} \Theta_{\alpha \gamma}\right) \\
-\Psi^{2} \Phi^{-2}\left(\Psi_{0}-a_{0}\right)\left(\eta_{\alpha \gamma}-\Psi^{-2} K_{\alpha \gamma}\right)-\left(\Phi_{\alpha}-b_{\alpha}\right) d_{\gamma} \\
-\frac{1}{2}\left(\Psi^{2} a_{\alpha} \Phi^{-2}-d_{\alpha}\right) c_{\gamma}, \\
-\left(\eta_{\alpha \mu}-\Psi^{-2} K_{\alpha \mu}\right) \Gamma_{\gamma 4}^{\mu}+\frac{1}{4}\left(a_{\alpha}-\Phi^{2} d_{\alpha} \Psi^{-2}\right)\left(3 d_{\gamma}+\Psi^{2} a_{\gamma} \Phi^{-2}\right) \\
+\left(\Psi_{\alpha}-c_{\alpha}\right)\left(\Psi_{\gamma}-c_{\gamma}\right)-\left(\omega_{\alpha \gamma}+\Phi^{-2} \Theta_{\alpha \gamma}\right)\left(\Psi_{0}-a_{0}\right) \\
+\Psi_{4}\left(\eta_{\alpha \gamma}-\Psi^{-2} K_{\alpha \gamma}\right),
\end{gathered}
$$

and

(a) $\bar{R}_{\alpha 40}^{\mu}=R_{\alpha 40}^{\mu}+\frac{1}{2}\left\{\left(\Psi^{2} a_{\alpha}-\Phi^{2} d_{\alpha}\right)\left(\Phi^{\mu}-\Psi^{\mu}+c^{\mu}-b^{\mu}\right)\right.$

$$
\left.+\left(\Psi_{\alpha}-\Phi_{\alpha}+b_{\alpha}-c_{\alpha}\right)\left(\Psi^{2} a^{\mu}-\Phi^{2} d^{\mu}\right)\right\},
$$

(b) $\bar{R}_{\alpha 40}^{0}=\frac{1}{2}\left\{\left(\Psi^{2} a_{\alpha} \Phi^{-2}-d_{\alpha}\right)_{\mid 0}+\left(\Psi^{2} a_{\alpha} \Phi^{-2}-d_{\alpha}\right)\left(\Phi_{0}-\Psi_{0}\right)\right\}$

$$
-\left(\Psi_{\alpha}-b_{\alpha}\right)_{\mid 4}+\Phi_{4}\left(\Psi_{\alpha}-\Phi_{\alpha}+b_{\alpha}-c_{\alpha}\right),
$$

(c) $\bar{R}_{\alpha 40}^{4}=\left(\Psi_{\alpha}-c_{\alpha}\right)_{\mid 0}-\frac{1}{2}\left\{\left(\Phi^{2} d_{\alpha} \Psi^{-2}-a_{\alpha}\right)_{\mid 4}\right.$

$$
\begin{aligned}
& \left.+\left(\Phi_{4}+\Psi_{4}\right)\left(\Phi^{2} d_{\alpha} \Psi^{-2}-a_{\alpha}\right)\right\} \\
& +\left(\Psi_{0}-a_{0}\right)\left(\Psi_{\alpha}-\Phi_{\alpha}+b_{\alpha}-c_{\alpha}\right) .
\end{aligned}
$$

Next, by using the local components for $\bar{R}$ and $R$ of type $(0,4)$ from $\left(A_{9}\right)$ and $\left(A_{10}\right)$, and taking into account $(3.5),(3.6),(3.7)$, and (3.8), we infer than

(a) $\bar{R}_{\alpha \beta \gamma \nu}=R_{\alpha \beta \gamma \nu}+\mathcal{A}_{(\gamma \nu)}\left\{\Phi^{-2}\left(\Theta_{\alpha \gamma}+\Phi^{2} \omega_{\alpha \gamma}\right)\left(\Theta_{\beta \nu}+\Phi^{2} \omega_{\beta \nu}\right)\right.$

$$
\left.-\Psi^{-2}\left(K_{\alpha \gamma}-\Psi^{2} \eta_{\alpha \gamma}\right)\left(K_{\beta \nu}-\Psi^{2} \eta_{\beta \nu}\right)\right\},
$$

(b) $\bar{R}_{\alpha 0 \gamma \nu}=\mathcal{A}_{(\gamma \nu)}\left\{\Theta_{\alpha \nu \mid \gamma}+\Theta_{\alpha \gamma} \Phi_{\nu}+\Phi^{2}\left(\omega_{\alpha \nu \mid \gamma}+\omega_{\alpha \nu} \Phi_{\gamma}\right)\right.$

$$
\begin{array}{r}
\left.+\frac{1}{2}\left(\Psi^{2} a_{\gamma}+\Phi^{2} d_{\gamma}\right)\left(\eta_{\alpha \nu}-\Psi^{-2} K_{\alpha \nu}\right)\right\} \\
+2 \Phi^{2}\left(\Phi_{\alpha}-b_{\alpha}\right) \omega_{\gamma \nu}+\left(\Psi^{2} a_{\alpha}-\Phi^{2} d_{\alpha}\right) \eta_{\gamma \nu},
\end{array}
$$

(c) $\bar{R}_{\alpha 4 \gamma \nu}=\mathcal{A}_{(\gamma \nu)}\left\{K_{\alpha \nu \mid \gamma}+K_{\alpha \gamma} \Psi_{\nu}+\Psi^{2}\left(\eta_{\alpha \gamma \mid \nu}+\eta_{\alpha \gamma} \Psi_{\nu}\right)+\right.$

$$
\begin{array}{r}
\left.+\frac{1}{2}\left(\Psi^{2} a_{\nu}+\Phi^{2} d_{\nu}\right)\left(\omega_{\alpha \gamma}+\Psi^{-2} \Theta_{\alpha \gamma}\right)\right\} \\
-2 \Psi^{2}\left(\Psi_{\alpha}-c_{\alpha}\right) \eta_{\gamma \nu}+\left(\Psi^{2} a_{\alpha}-\Phi^{2} d_{\alpha}\right) \omega_{\gamma \nu},
\end{array}
$$




$$
\begin{aligned}
& \text { (a) } \bar{R}_{\alpha \beta 0 \gamma}=R_{\alpha \beta 0 \gamma}+\mathcal{A}_{(\alpha \beta)}\left\{\left(\Phi_{\alpha}-b_{\alpha}\right)\left(\Theta_{\beta \gamma}+\Phi^{2} \omega_{\beta \gamma}\right)\right. \\
& \left.+\frac{1}{2}\left(\Phi^{2} d_{\alpha} \Psi^{-2}-a_{\alpha}\right)\left(K_{\beta \gamma}-\Psi^{2} \eta_{\beta \gamma}\right)\right\}, \\
& \text { (b) } \bar{R}_{\alpha 00 \gamma}=\Phi_{0}\left(\Theta_{\alpha \gamma}+\Phi^{2} \omega_{\alpha \gamma}\right)+\left(\Theta_{\alpha \nu}+\Phi^{2} \omega_{\alpha \nu}\right)\left(\Theta_{\alpha}^{\nu}+\Phi^{2} \omega_{\alpha}^{\nu}\right) \\
& +\Phi^{2}\left\{b_{\alpha \mid \gamma}-\Phi_{\alpha \mid \gamma}+\left(\omega_{\alpha \gamma}+\Phi^{-2} \Theta_{\alpha \gamma}\right)_{\mid 0}-\left(\Phi_{\alpha}-b_{\alpha}\right)\left(\Phi_{\gamma}-b_{\gamma}\right)\right. \\
& +\Phi_{4}\left(\eta_{\alpha \gamma}-\Psi^{-2} K_{\alpha \gamma}\right)-\frac{1}{4}\left(3 d_{\alpha} a_{\gamma}-a_{\alpha} d_{\gamma}+\Phi^{2} d_{\alpha} d_{\gamma} \Psi^{-2}\right. \\
& \left.\left.-3 \Psi^{2} a_{\alpha} a_{\gamma} \Phi^{-2}\right)\right\}, \\
& \text { (c) } \bar{R}_{\alpha 40 \gamma}=\Psi^{2}\left\{\frac{1}{2}\left(\Phi^{2} d_{\alpha} \Psi^{-2}-a_{\alpha}\right)_{\mid \gamma}-\left(\eta_{\alpha \gamma}-\Psi^{-2} K_{\alpha \gamma}\right)_{\mid 0}\right. \\
& \left.-\left(\Psi_{\alpha}-c_{\alpha}\right) a_{\gamma}\right\}+\left(K_{\alpha \nu}-\Psi^{2} \eta_{\alpha \nu}\right)\left(\Theta_{\gamma}^{\nu}+\Phi^{2} \omega_{\gamma}^{\nu}\right)+ \\
& \frac{1}{2}\left\{\left(\Phi_{\alpha}-b_{\alpha}\right)\left(\Phi^{2} d_{\gamma}+\Psi^{2} a_{\gamma}\right)+\left(\Psi_{\gamma}-c_{\gamma}\right)\left(\Phi^{2} d_{\alpha}-\Psi^{2} a_{\alpha}\right)\right\} \\
& -\Phi_{4}\left(\Theta_{\alpha \gamma}+\Phi^{2} \omega_{\alpha \gamma}\right)+\Psi_{0}\left(K_{\alpha \gamma}-\Psi^{2} \eta_{\alpha \gamma}\right), \\
& \text { (a) } \bar{R}_{\alpha \beta 4 \gamma}=R_{\alpha \beta 4 \gamma}+\mathcal{A}_{(\alpha \beta)}\left\{\frac{1}{2}\left(\Psi^{2} a_{\alpha} \Phi^{-2}-d_{\alpha}\right)\left(\Theta_{\beta \gamma}+\Phi^{2} \omega_{\beta \gamma}\right)\right. \\
& \left.+\left(\Psi_{\alpha}-c_{\alpha}\right)\left(K_{\beta \gamma}-\Psi^{2} \eta_{\beta \gamma}\right)\right\}, \\
& \text { (b) } \bar{R}_{\alpha 04 \gamma}=\Phi^{2}\left\{\left(\omega_{\alpha \gamma}+\Phi^{-2} \Theta_{\alpha \gamma}\right)_{\mid 4}-\frac{1}{2}\left(\Psi^{2} a_{\alpha} \Phi^{-2}-d_{\alpha}\right)_{\mid \gamma}\right. \\
& \left.+\left(\Phi_{\alpha}-b_{\alpha}\right) d_{\gamma}\right\}+\left(\Theta_{\alpha \nu}+\Phi^{2} \omega_{\alpha \nu}\right)\left(K_{\gamma}^{\nu}-\Psi^{2} \eta_{\gamma}^{\nu}\right) \\
& -\frac{1}{2}\left\{\left(\Psi^{2} a_{\alpha}-\Phi^{2} d_{\alpha}\right)\left(\Psi_{\gamma}-c_{\gamma}\right)\right. \\
& \left.+\left(\Psi^{2} a_{\gamma}+\Phi^{2} d_{\gamma}\right)\left(\Psi_{\alpha}-c_{\alpha}\right)\right\}+\Phi_{4}\left(\Theta_{\alpha \gamma}+\Phi^{2} \omega_{\alpha \gamma}\right) \\
& +\left(\Psi_{0}-a_{0}\right)\left(\Psi^{-2} \eta_{\alpha \gamma}-K_{\alpha \gamma}\right), \\
& \text { (c) } \bar{R}_{\alpha 44 \gamma}=\Psi^{2}\left\{\left(\Psi_{\alpha}-c_{\alpha}\right)_{\mid \gamma}-\left(\eta_{\alpha \gamma}-\Psi^{-2} K_{\alpha \gamma}\right)_{\mid 4}\right. \\
& \left.+\left(\Psi_{\alpha}-c_{\alpha}\right)\left(\Psi_{\gamma}-c_{\gamma}\right)-\left(\Psi_{0}-a_{0}\right)\left(\omega_{\alpha \gamma}+\Phi^{-2} \Theta_{\alpha \gamma}\right)\right\} \\
& +\left(K_{\alpha \nu}-\Psi^{2} \eta_{\alpha \nu}\right)\left(K_{\gamma}^{\nu}-\Psi^{2} \eta_{\gamma}^{\nu}\right)+\frac{1}{4}\left\{3 \Psi^{2} a_{\alpha} d_{\gamma}-\Psi^{2} a_{\gamma} d_{\alpha}\right. \\
& \left.-3 \Phi^{2} d_{\alpha} d_{\gamma}+\Psi^{4} a_{\alpha} a_{\gamma} \Phi^{-2}\right\}-\Psi_{4}\left(K_{\alpha \gamma}-\Psi^{2} \eta_{\alpha \gamma}\right),
\end{aligned}
$$

and

$$
\begin{gathered}
(a) \quad \bar{R}_{\alpha \beta 40}=R_{\alpha \beta 40} \\
+\frac{1}{2} \mathcal{A}_{(\alpha \beta)}\left\{\left(\Psi^{2} a_{\alpha}-\Phi^{2} d_{\alpha}\right)\left(\Phi_{\beta}-\Psi_{\beta}+c_{\beta}-b_{\beta}\right)\right\}, \\
(b) \quad \bar{R}_{\alpha 040}=\Phi^{2}\left\{\left(\Phi_{\alpha}-b_{\alpha}\right)_{\mid 4}+\Phi_{4}\left(\Phi_{\alpha}-\Psi_{\alpha}+c_{\alpha}-b_{\alpha}\right)\right. \\
\left.+\frac{1}{2}\left(d_{\alpha}-\Psi^{2} a_{\alpha} \Phi^{-2}\right)_{\mid 0}\right\}+\frac{1}{2}\left(\Phi^{2} d_{\alpha}-\Psi^{2} a_{\alpha}\right)\left(\Phi_{0}-a_{0}\right), \\
(c) \quad \bar{R}_{\alpha 440}=\Psi^{2}\left\{\left(\Psi_{\alpha}-c_{\alpha}\right)_{\mid 0}+\frac{1}{2}\left(a_{\alpha}-\Phi^{2} d_{\alpha} \Psi^{-2}\right)_{\mid 4}\right. \\
\left.-\left(\Psi_{0}-a_{0}\right)\left(\Phi_{\alpha}-\Psi_{\alpha}+c_{\alpha}-b_{\alpha}\right)\right\} \\
+\frac{1}{2}\left(\Phi^{2} d_{\alpha}-\Psi^{2} a_{\alpha}\right)\left(\Phi_{4}+\Psi_{4}\right) .
\end{gathered}
$$

The equations (3.9) - (3.12) are called structure equations for the spatial distribution with respect to the $(1+1+3)$ threading of the $5 D$ universe $(\bar{M}, \bar{g})$. Moreover, in analogy with theory of the submanifolds, we call $\{(3.9 a)\}$, $\{(3.9 b),(3.9 c),(3.10 a),(3.11 a)\}$ and $\{(3.10 b),(3.10 c),(3.11 b),(3.11 c),(3.12)\}$, the Gauss equations, Codazzi equations and Ricci equations respectively, for the spatial distribution. 
Now, taking into account the symmetries of $\bar{R}$ we deduce some important identities for the curvature tensor field of the Riemannian spatial connection and for the kinematic quantities of $(\bar{M}, \bar{g})$. First, from (3.9a) we obtain

$$
\begin{gathered}
\text { (a) } R_{\alpha \beta \gamma \nu}+R_{\alpha \beta \nu \gamma}=0, \quad \text { (b) } R_{\alpha \beta \gamma \nu}+R_{\beta \alpha \gamma \nu}=0 \\
\text { (c) } R_{\alpha \beta \gamma \nu}- \\
R_{\gamma \nu \alpha \beta}=2 \mathcal{A}_{(\gamma \nu)}\left\{\omega_{\gamma \alpha} \Theta_{\nu \beta}+\omega_{\nu \beta} \Theta_{\gamma \alpha}\right. \\
\left.+\eta_{\gamma \alpha} K_{\nu \beta}+\eta_{\nu \beta} K_{\gamma \alpha}\right\}
\end{gathered}
$$

Then, taking into account that

$$
\bar{R}_{\alpha 0 \gamma \nu}=-\bar{R}_{\gamma \nu 0 \alpha}
$$

and using (3.9b) and (3.10a), we infer that

$$
\begin{aligned}
R_{\gamma \nu 0 \alpha}= & \mathcal{A}_{(\nu \gamma)}\left\{\Psi^{2}\left(b_{\gamma} \omega_{\nu \alpha}+\omega_{\nu \alpha \mid \gamma}+d_{\gamma} \eta_{\nu \alpha}\right)+\Theta_{\nu \alpha \mid \gamma}+a_{\gamma} K_{\alpha \nu}\right. \\
& \left.+b_{\gamma} \Theta_{\alpha \nu}\right\}+2 \Phi^{2}\left(\Phi_{\alpha}-b_{\alpha}\right) \omega_{\nu \gamma}+\left(\Psi^{2} a_{\alpha}-\Phi^{2} d_{\alpha}\right) \eta_{\nu \gamma} .
\end{aligned}
$$

Similarly, from (3.9c) and (3.11a), we deduce that

$$
\begin{gathered}
R_{\gamma \nu 0 \alpha}=\mathcal{A}_{(\nu \gamma)}\left\{K_{\alpha \nu \mid \gamma}+\Psi^{2}\left(\eta_{\alpha \gamma \mid \nu}+2 \eta_{\alpha \gamma} \Psi_{\nu}\right)\right. \\
+\frac{1}{2}\left(\left(\Phi^{2} d_{\nu}+\Psi^{2} a_{\nu}\right) \omega_{\alpha \gamma}+\left(\Psi^{2} a_{\nu}-\Phi^{2} d_{\nu}+2 \Psi^{2} a_{\nu} \Phi^{-2}\right) \Theta_{\alpha \gamma}\right) \\
\left.+c_{\gamma}\left(K_{\nu \alpha}-\Psi^{2} \eta_{\nu \alpha}\right)\right\}-2 \Psi^{2}\left(\Psi_{\alpha}-c_{\alpha}\right) \eta_{\gamma \nu}+\left(\Psi^{2} a_{\alpha}-\Phi^{2} d_{\alpha}\right) \omega_{\gamma \nu} .
\end{gathered}
$$

Finally, taking into account that both $\bar{R}_{\alpha 0 \gamma 0}$ and $\bar{R}_{\alpha 4 \gamma 4}$ are symmetric tensor fields with respect to the indices $(\alpha \gamma)$, from $(3.10 \mathrm{~b})$ and $(3.11 \mathrm{c})$ we obtain

$$
\begin{array}{r}
\bar{R}_{\alpha 0 \gamma 0}=-\Phi_{0} \Theta_{\alpha \gamma}-\Theta_{\alpha \nu} \Theta_{\gamma}^{\nu}-\Phi^{4} \omega_{\alpha \nu} \omega_{\gamma}^{\nu}-\Phi^{2}\left\{\frac { 1 } { 2 } \left(b_{\alpha \mid \gamma}+b_{\gamma \mid \alpha}\right.\right. \\
\left.-\Phi_{\alpha \mid \gamma}-\Phi_{\gamma \mid \alpha}\right)+\left(\Phi^{-2} \Theta_{\alpha \gamma}\right) \mid 0-\left(\Phi_{\alpha}-b_{\alpha}\right)\left(\Phi_{\gamma}-b_{\gamma}\right) \\
-\Phi_{4} \Psi^{-2} K_{\alpha \gamma}-\frac{1}{4}\left(a_{\alpha} d_{\gamma}+a_{\gamma} d_{\alpha}\right. \\
\left.\left.+\Phi^{2} d_{\gamma} d_{\alpha} \Psi^{-2}-3 \Psi^{2} a_{\gamma} a_{\alpha} \Phi^{-2}\right)\right\},
\end{array}
$$

and

$$
\begin{gathered}
\bar{R}_{\alpha 4 \gamma 4}=\Psi_{4} K_{\alpha \gamma}-K_{\alpha \nu} K_{\gamma}^{\nu}-\Psi^{4} \eta_{\alpha \nu} \eta_{\gamma}^{\nu}-\Psi^{2}\left\{\frac { 1 } { 2 } \left(\Psi_{\alpha \mid \gamma}+\Psi_{\gamma \mid \alpha}\right.\right. \\
\left.-c_{\alpha \mid \gamma}-c_{\gamma \mid \alpha}\right)+\left(\Psi^{-2} K_{\alpha \gamma}\right)_{\mid 4}+\left(\Psi_{\alpha}-c_{\alpha}\right)\left(\Psi_{\gamma}-c_{\gamma}\right) \\
-\left(\Psi_{0}-a_{0}\right) \Phi^{-2} \Theta_{\alpha \gamma}-\frac{1}{4}\left(a_{\alpha} d_{\gamma}+a_{\gamma} d_{\alpha}\right. \\
\left.\left.+\Psi^{2} a_{\alpha} a_{\gamma} \Psi^{-2}-3 \Phi^{2} d_{\alpha} d_{\gamma} \Psi^{-2}\right)\right\}
\end{gathered}
$$

\section{Ricci tensor and scalar curvature of a $5 D$ universe}

Let $(\bar{M}, \bar{g})$ be a $5 D$ universe and $\left\{E_{\alpha}\right\}$ be an orthonormal basis in $\Gamma(\mathcal{S} \bar{M})$. Then $\left\{\Phi^{-1} \frac{\delta}{\delta x^{0}}, E_{\alpha}, \Psi^{-1} \frac{\partial}{\partial x^{4}}\right\}$ is an orthonormal frame field locally defined on $\bar{M}$. Thus the Ricci tensor of $(\bar{M}, \bar{g})$ is given by

$$
\begin{gathered}
\overline{\operatorname{Ric}}(X, Y)=-\Phi^{-2} \bar{R}\left(\frac{\delta}{\delta x^{0}}, X, \frac{\delta}{\delta x^{0}}, Y\right)+\sum_{\alpha=1}^{3}\left\{\bar{R}\left(E_{\alpha}, X, E_{\alpha}, Y\right)\right\} \\
+\Psi^{-2} \bar{R}\left(\frac{\partial}{\partial x^{4}}, X, \frac{\partial}{\partial x^{4}}, Y\right),
\end{gathered}
$$

for all $X, Y \in \Gamma(T \bar{M})$. Now, we put

$$
E_{\alpha}=E_{\alpha}^{\gamma} \frac{\delta}{\delta x^{\gamma}}
$$


and obtain

$$
h^{\gamma \mu}=\sum_{\alpha=1}^{3} E_{\alpha}^{\gamma} E_{\alpha}^{\mu} .
$$

For local components of Ric it is more convenient to use the adapted frame field $\left\{\frac{\delta}{\delta x^{0}}, \frac{\delta}{\delta x^{\alpha}}, \frac{\partial}{\partial x^{4}}\right\}$, instead of the above orthonormal frame field. Thus we have
(a) $\bar{R}_{\alpha \beta}=\overline{\operatorname{Ric}}\left(\frac{\delta}{\delta x^{\beta}}, \frac{\delta}{\delta x^{\alpha}}\right), \quad$ (b) $\bar{R}_{\alpha 0}=\overline{\operatorname{Ric}}\left(\frac{\delta}{\delta x^{0}}, \frac{\delta}{\delta x^{\alpha}}\right)$,
(c) $\bar{R}_{00}=\operatorname{Ric}\left(\frac{\delta}{\delta x^{0}}, \frac{\delta}{\delta x^{0}}\right), \quad(d) \quad \bar{R}_{\alpha 4}=\operatorname{Ric}\left(\frac{\partial}{\partial x^{4}}, \frac{\delta}{\delta x^{\alpha}}\right)$,
(e) $\bar{R}_{44}=\overline{\operatorname{Ric}}\left(\frac{\partial}{\partial x^{4}}, \frac{\partial}{\partial x^{4}}\right), \quad(f) \quad \bar{R}_{40}=\overline{\operatorname{Ri}}\left(\frac{\delta}{\delta x^{0}}, \frac{\partial}{\partial x^{4}}\right)$.

By using (4.1) and $\left(A_{9}\right)$ into (4.4), we obtain
(a) $\bar{R}_{\alpha \beta}=-\Phi^{-2} \bar{R}_{\alpha 0 \beta 0}+h^{\mu \gamma} \bar{R}_{\alpha \mu \beta \gamma}+\Psi^{-2} \bar{R}_{\alpha 4 \beta 4}$,
(b) $\bar{R}_{\alpha 0}=h^{\mu \gamma} \bar{R}_{\alpha \mu 0 \gamma}+\Psi^{-2} \bar{R}_{\alpha 404}$,
(c) $\bar{R}_{00}=h^{\mu \gamma} \bar{R}_{0 \mu 0 \gamma}+\Psi^{-2} \bar{R}_{0404}$,
(d) $\bar{R}_{\alpha 4}=-\Phi^{-2} \bar{R}_{\alpha 040}+h^{\mu \gamma} \bar{R}_{\alpha \mu 4 \gamma}$,
(e) $\quad \bar{R}_{44}=-\Phi^{-2} \bar{R}_{4040}+h^{\mu \gamma} \bar{R}_{4 \mu 4 \gamma}$,
(f) $\quad \bar{R}_{40}=h^{\mu \gamma} \bar{R}_{4 \mu 0 \gamma}$.

Next, by some long calculations using [(3.9) - (3.12)] into (4.5), we deduce that 


$$
\begin{aligned}
& \text { (a) } \bar{R}_{\alpha \beta}=R_{\alpha \beta}+\frac{1}{2}\left\{\left(b_{\alpha}+c_{\alpha}-\Phi_{\alpha}-\Psi_{\alpha}\right)_{\mid \beta}+\left(b_{\beta}+c_{\beta}\right.\right. \\
& \left.\left.-\Phi_{\beta}-\Psi_{\beta}\right)_{\mid \alpha}\right\}+\left(\Phi^{-2} \Theta_{\alpha \beta}\right)_{\mid 0}-\left(\Psi^{-2} K_{\alpha \beta}\right)_{\mid 4} \\
& +\Phi^{-2}\left(\Phi_{0}+\Psi_{0}-a_{0}+\Theta\right) \Theta_{\alpha \beta}+\Psi^{-2}\left(\Psi_{4}-\Phi_{4}-K\right) K_{\alpha \beta} \\
& -\left(\Phi_{\alpha}-b_{\alpha}\right)\left(\Phi_{\beta}-b_{\beta}\right)-\left(\Psi_{\alpha}-c_{\alpha}\right)\left(\Psi_{\beta}-c_{\beta}\right), \\
& \text { (b) } \bar{R}_{\alpha 0}=R_{\alpha 0 \gamma}^{\gamma}+\left(c_{\alpha}-\Psi_{\alpha}\right)_{\mid 0}+\left(\Phi^{2} d_{\alpha} \Psi^{-2}-a_{\alpha}\right)_{\mid 4} \\
& -\left(\Psi_{0}-a_{0}\right)\left(\Psi_{\alpha}-c_{\alpha}\right)+\frac{1}{2}\left(K-\Phi_{4}-\Psi_{4}\right)\left(\Phi^{2} d_{\alpha} \Psi^{-2}-a_{\alpha}\right) \\
& +\left(\Theta+\Psi_{0}-a_{0}\right)\left(\Phi_{\alpha}-b_{\alpha}\right)-\left(\Phi^{\gamma}-b^{\gamma}\right)\left(\Theta_{\alpha \gamma}+\Phi^{2} \omega_{\alpha \gamma}\right) \\
& -\frac{1}{2}\left(\Phi^{2} d^{\gamma} \Psi^{-2}-a^{\gamma}\right)\left(K_{\alpha \gamma}-\Psi^{2} \eta_{\alpha \gamma}\right) \\
& \text { (c) } \quad \bar{R}_{00}=\left(c_{0}-\Psi_{0}-\Theta\right)_{\mid 0}+\left(\Phi^{2} \Phi_{4} \Psi^{-2}\right)_{\mid 4}+\Phi_{0} \Theta-\Theta_{\alpha}^{\gamma} \Theta_{\gamma}^{\alpha} \\
& +\left(\Psi_{0}-a_{0}\right)\left(\Phi_{0}-\Psi_{0}+a_{0}\right)+\Phi^{2}\left\{\Phi_{\mid \gamma}^{\gamma}-b_{\mid \gamma}^{\gamma}-\Phi^{2} \omega_{\alpha}^{\gamma} \omega_{\gamma}^{\alpha}\right. \\
& +\left(\Phi^{\gamma}-b^{\gamma}\right)\left(\Phi_{\gamma}-b_{\gamma}+c_{\gamma}-\Psi_{\gamma}\right)-K \Phi_{4} \Psi^{-2} \\
& \left.+\frac{1}{2}\left(\Phi^{2} d_{\gamma} d^{\gamma} \Psi^{-2}-\Psi^{2} a_{\gamma} a^{\gamma} \Phi^{-2}\right)\right\}, \\
& \text { (d) } \quad \bar{R}_{\alpha 4}=R_{\alpha 4 \gamma}^{\gamma}+\left(b_{\alpha}-\Phi_{\alpha}\right)_{\mid 4}+\frac{1}{2}\left(\Psi^{2} a_{\alpha} \Phi^{-2}-d_{\alpha}\right)_{\mid 0} \\
& +\left(K+\Phi_{4}\right)\left(\Psi_{\alpha}-c_{\alpha}\right)+\Phi_{4}\left(b_{\alpha}-\Phi_{\alpha}\right)+\frac{1}{2}\left\{\left(a_{0}-\Phi_{0}+\Theta\right) \Psi^{2} a_{\alpha} \Phi^{-2}\right. \\
& \left.+\left(a_{0}-\Phi_{0}-\Theta\right) d_{\alpha}-\left(\Psi^{2} a^{\gamma} \Phi^{-2}-d^{\gamma}\right)\left(\Theta_{\alpha \gamma}+\Phi^{2} \omega_{\alpha \gamma}\right)\right\} \\
& -\left(\Psi^{\gamma}-c^{\gamma}\right)\left(K_{\alpha \gamma}-\Psi^{2} \eta_{\alpha \gamma}\right), \\
& \text { (e) } \bar{R}_{44}=3 \Psi_{4} K-K_{\mid 4}-K_{\alpha}^{\gamma} K_{\gamma}^{\alpha}+\frac{1}{2} \Phi^{2} d_{\gamma} d^{\gamma}+\Psi^{2}\left\{c_{\mid \gamma}^{\gamma}-\Psi_{\mid \gamma}^{\gamma}\right. \\
& -\Psi^{2} \eta_{\alpha}^{\gamma} \eta_{\gamma}^{\alpha}+\Phi^{-2}\left(\Psi_{0}-a_{0}\right)\left(\Theta+\Psi_{0}-\Phi_{0}-a_{0}\right) \\
& \left.-\left(\Psi^{\gamma}-c^{\gamma}\right)\left(\Psi_{\gamma}+\Phi_{\gamma}-c_{\gamma}-b_{\gamma}\right)-\frac{1}{2} \Psi^{2} a_{\gamma} a^{\gamma} \Phi^{-2}\right\}, \\
& \text { (f) } \quad \bar{R}_{40}=3 \Phi_{4} \Theta+\left(a_{0}-\Psi_{0}\right) K-\Theta_{\mid 4}+\Psi^{2} a_{\gamma}\left(\Psi^{\gamma}-c^{\gamma}\right) \\
& -\left(\Theta_{\alpha}^{\gamma}+\Phi^{2} \omega_{\alpha}^{\gamma}\right)\left(K_{\gamma}^{\alpha}-\Psi^{2} \eta_{\gamma}^{\alpha}\right)+\Phi^{2}\left\{d_{\gamma}\left(\Phi^{\gamma}-b^{\gamma}\right)\right. \\
& \left.+\frac{1}{2}\left(\Psi^{2} a^{\gamma} \Phi^{-2}-d^{\gamma}\right)_{\mid \gamma}\right\},
\end{aligned}
$$

where we put

$$
R_{\alpha \beta}=\frac{1}{2}\left(R_{\alpha \beta \gamma}^{\gamma}+R_{\beta \alpha \gamma}^{\gamma}\right) .
$$

The spatial tensor field $R_{\alpha \beta}$ is called the spatial Ricci tensor of the $5 D$ universe $(\bar{M}, \bar{g})$.

Finally, we obtain a formula for the scalar curvature $\bar{R}$ in terms of spatial tensor fields. First, we have

$$
\bar{R}=-\Phi^{-2} \bar{R}_{00}+h^{\gamma \nu} \bar{R}_{\gamma \nu}+\Psi^{-2} \bar{R}_{44} .
$$

Then by direct calculations using (4.5a), (4.5c) and (4.5e) into (4.8), we obtain 


$$
\begin{aligned}
&(a) \bar{R}=R+2\left\{\left(b^{\gamma}+c^{\gamma}-\Phi^{\gamma}-\Psi^{\gamma}\right)_{\mid \gamma}-\left(\Phi^{\gamma}-b^{\gamma}\right)\left(\Phi_{\gamma}-b_{\gamma}\right)\right. \\
&\left.-\left(\Psi^{\gamma}-c^{\gamma}\right)\left(\Psi_{\gamma}-c_{\gamma}\right)\right\}+\Phi^{2} \omega_{\alpha}^{\gamma} \omega_{\gamma}^{\alpha}-\Psi^{2} \eta_{\alpha}^{\gamma} \eta_{\gamma}^{\alpha} \\
&+\Phi^{-2}\left\{\Theta^{2}-2 \Phi_{0} \Theta+2\left(\Psi_{0}-a_{0}\right)\left(\Theta+\Psi_{0}-a_{0}-\Phi_{0}\right)\right. \\
&\left.+\left(\Psi_{0}-a_{0}\right)_{\mid 0}+2 \Theta_{\mid 0}-\left(\Phi^{2} \Phi_{4} \Psi^{-2}\right)_{\mid 4}+\Theta_{\alpha}^{\gamma} \Theta_{\gamma}^{\alpha}\right\} \\
&-\Psi^{-2}\left\{K^{2}+K_{\mid 4}+\left(\Phi_{4}-7 \Psi_{4}\right) K+K_{\alpha}^{\gamma} K_{\gamma}^{\alpha}\right\},
\end{aligned}
$$

where we put

$$
R=h^{\gamma \mu} R_{\gamma \mu}
$$

We call $R$ the spatial scalar curvature of the $5 D$ universe $(\bar{M}, \bar{g})$. It is important to mention that $R_{\alpha \beta}$ and $R$ characterize the geometry of the spatial distribution, which in our study is not necessarily integrable.

\section{Splitting of the Einstein field equations of $(\bar{M}, \bar{g})$}

In the first part of this section we obtain the local components of the Einstein gravitational tensor field $\bar{G}$ of $(\bar{M}, \bar{g})$ with respect to the adapted frame field $\left\{\frac{\delta}{\delta x^{0}}, \frac{\delta}{\delta x^{\alpha}}, \frac{\partial}{\partial x^{4}},\right\}$. We start with the coordinate-free form of $\bar{G}$ given by

$$
\bar{G}(X, Y)=\overline{R i c}(X, Y)-\frac{\bar{R}}{2} \bar{g}(X, Y), \quad \forall X, Y \in \Gamma(T \bar{M}) .
$$

Then we have the following local components:
(a) $\bar{G}_{\alpha \beta}=\bar{G}\left(\frac{\delta}{\delta x^{\beta}}, \frac{\delta}{\delta x^{\alpha}}\right)=\bar{R}_{\alpha \beta}-\frac{\bar{R}}{2} h_{\alpha \beta}$,
(b) $\bar{G}_{\alpha 0}=\bar{G}\left(\frac{\delta}{\delta x^{0}}, \frac{\delta}{\delta x^{\alpha}}\right)=\bar{R}_{\alpha 0}$,
(c) $\bar{G}_{00}=\bar{G}\left(\frac{\delta}{\delta x^{0}}, \frac{\delta}{\delta x^{0}}\right)=\bar{R}_{00}+\frac{\bar{R}}{2} \Phi^{2}$,
(d) $\bar{G}_{\alpha 4}=\bar{G}\left(\frac{\partial}{\partial x^{4}}, \frac{\delta}{\delta x^{\alpha}}\right)=\bar{R}_{\alpha 4}$,
(e) $\bar{G}_{44}=\bar{G}\left(\frac{\partial}{\partial x^{4}}, \frac{\partial}{\partial x^{4}}\right)=\bar{R}_{44}-\frac{\bar{R}}{2} \Psi^{2}$,
(f) $\bar{G}_{40}=\bar{G}\left(\frac{\delta}{\delta x^{0}}, \frac{\partial}{\partial x^{4}}\right)=\bar{R}_{40}$.

Next, by using (4.6) and (4.9) into (5.2), we obtain 


$$
\begin{aligned}
& \text { (a) } \bar{G}_{\alpha \beta}=G_{\alpha \beta}+\frac{1}{2}\left\{\left(b_{\alpha}+c_{\alpha}-\Phi_{\alpha}-\Psi_{\alpha}\right)_{\mid \beta}+\left(b_{\beta}+c_{\beta}\right.\right. \\
& \left.\left.-\Phi_{\beta}-\Psi_{\beta}\right)_{\mid \alpha}\right\}+\left(\Phi^{-2} \Theta_{\alpha \beta}\right)_{\mid 0}-\left(\Psi^{-2} K_{\alpha \beta}\right)_{\mid 4} \\
& +\Phi^{-2}\left(\Phi_{0}+\Psi_{0}-a_{0}+\Theta\right) \Theta_{\alpha \beta}+\Psi^{-2}\left(\Psi_{4}-\Phi_{4}-K\right) K_{\alpha \beta} \\
& +\left(\Phi_{\alpha}-b_{\alpha}\right)\left(\Phi_{\beta}-b_{\beta}\right)-\left(\Psi_{\alpha}-c_{\alpha}\right)\left(\Psi_{\beta}-c_{\beta}\right) \\
& -\frac{1}{2}\left\{\Phi^{2} \omega_{\mu}^{\gamma} \omega_{\gamma}^{\mu}-\Psi^{2} \eta_{\alpha}^{\gamma} \eta_{\gamma}^{\alpha}+2\left(\left(b^{\gamma}+c^{\gamma}-\Phi^{\gamma}-\Psi^{\gamma}\right)_{\mid \gamma}\right.\right. \\
& \left.-\left(\Phi^{\gamma}-b^{\gamma}\right)\left(\Phi_{\gamma}-b_{\gamma}\right)-\left(\Psi^{\gamma}-c^{\gamma}\right)\left(\Psi_{\gamma}-c_{\gamma}\right)\right) \\
& +\Phi^{-2}\left(\Theta^{2}-2 \Phi_{0} \Theta+2\left(\Psi_{0}-a_{0}\right)\left(\Theta+\Psi_{0}-a_{0}-\Phi_{0}\right)\right. \\
& \left.+\left(\Psi_{0}-a_{0}\right)_{\mid 0}+2 \Theta_{\mid 0}-\left(\Phi^{2} \Phi_{4} \Psi^{-2}\right)_{\mid 4}+\Theta_{\mu}^{\gamma} \Theta_{\gamma}^{\mu}\right) \\
& \left.-\Psi^{-2}\left(K^{2}+\left(\Phi_{4}-7 \Psi_{4}\right) K+K_{\mid 4}+K_{\mu}^{\gamma} K_{\gamma}^{\mu}\right)\right\} h_{\alpha \beta}, \\
& \text { (b) } \bar{G}_{\alpha 0}=R_{\alpha 0 \gamma}^{\gamma}+\left(c_{\alpha}-\Psi_{\alpha}\right)_{\mid 0}+\left(\Phi^{2} d_{\alpha} \Psi^{-2}-a_{\alpha}\right)_{\mid 4} \\
& -\left(\Psi_{0}-a_{0}\right)\left(\Psi_{\alpha}-c_{\alpha}\right)+\left(\Theta+\Psi_{0}-a_{0}\right)\left(\Phi_{\alpha}-b_{\alpha}\right) \\
& -\left(\Phi^{\gamma}-b^{\gamma}\right)\left(\Theta_{\alpha \gamma}+\Phi^{2} \omega_{\alpha \gamma}\right)+\frac{1}{2}\left\{\left(K-\Phi_{4}-\Psi_{4}\right)\left(\Phi^{2} d_{\alpha} \Psi^{-2}-a_{\alpha}\right)\right. \\
& \left.-\left(\Phi^{2} d^{\gamma} \Psi^{-2}-a^{\gamma}\right)\left(K_{\alpha \gamma}-\Psi^{2} \eta_{\alpha \gamma}\right)\right\} \\
& \text { (c) } \quad \bar{G}_{00}=\frac{1}{2}\left\{\Theta^{2}+\left(a_{0}-\Psi_{0}\right)_{\mid 0}+\left(\Phi^{2} \Phi_{4} \Psi^{-2}\right)_{\mid 4}+2\left(\Psi_{0}-a_{0}\right) \Theta\right. \\
& +\Phi^{2}\left(R+\Phi^{2}\left(d^{\gamma} d_{\gamma} \Psi^{-2}-\omega_{\alpha}^{\gamma} \omega_{\gamma}^{\alpha}\right)-\Psi^{2}\left(a^{\gamma} a_{\gamma} \Phi^{-2}+\eta_{\alpha}^{\gamma} \eta_{\gamma}^{\alpha}\right)\right. \\
& -2\left(\Psi_{\gamma}-c_{\gamma}\right)\left(\Phi^{\gamma}+\Psi^{\gamma}-b^{\gamma}-c^{\gamma}\right)-2 K \Phi_{4}+2 c_{\mid \gamma}^{\gamma}-2 \Psi_{\mid \gamma}^{\gamma} \\
& \left.\left.-\Psi^{-2}\left(K^{2}+\left(\Phi_{4}-7 \Psi_{4}\right) K+K_{\mid 4}+K_{\alpha}^{\gamma} K_{\gamma}^{\alpha}\right)\right)\right\}, \\
& \text { (d) } \bar{G}_{\alpha 4}=R_{\alpha 4 \gamma}^{\gamma}+\left(b_{\alpha}-\Phi_{\alpha}\right)_{\mid 4}+\frac{1}{2}\left(\Psi^{2} a_{\alpha} \Phi^{-2}-d_{\alpha}\right)_{\mid 0} \\
& +\left(K+\Phi_{4}\right)\left(\Psi_{\alpha}-c_{\alpha}\right)+\Phi_{4}\left(b_{\alpha}-\Phi_{\alpha}\right)+\frac{1}{2}\left\{\left(a_{0}-\Phi_{0}+\Theta\right) \Psi^{2} a_{\alpha} \Phi^{-2}\right. \\
& \left.+\left(a_{0}-\Phi_{0}-\Theta\right) d_{\alpha}-\left(\Psi^{2} a^{\gamma} \Phi^{-2}-d^{\gamma}\right)\left(\Theta_{\alpha \gamma}+\Phi^{2} \omega_{\alpha \gamma}\right)\right\} \\
& -\left(\Psi^{\gamma}-c^{\gamma}\right)\left(K_{\alpha \gamma}-\Psi^{2} \eta_{\alpha \gamma}\right), \\
& \text { (e) } \bar{G}_{44}=\frac{1}{2}\left\{K^{2}+\left(\Phi_{4}-\Psi_{4}\right) K-K_{\mid 4}-K_{\alpha}^{\gamma} K_{\gamma}^{\alpha}+\Phi^{2} d_{\gamma} d^{\gamma}\right\} \\
& +\Psi^{2}\left\{\left(\Phi^{\gamma}-\Psi^{\gamma}+c^{\gamma}-b^{\gamma}\right)\left(\Phi_{\gamma}-b_{\gamma}\right)-\frac{1}{2}\left(\Phi^{2} \omega_{\alpha}^{\gamma} \omega_{\gamma}^{\alpha}+\Psi^{2} \eta_{\alpha}^{\gamma} \eta_{\gamma}^{\alpha}\right.\right. \\
& \left.+\Psi^{2} a_{\gamma} a^{\gamma} \Phi^{-2}+R\right)+\Phi_{\mid \gamma}^{\gamma}-b_{\mid \gamma}^{\gamma}-\Psi^{-2}\left(\Theta_{\mid 0}-\Phi_{0} \Theta\right. \\
& \left.\left.+\frac{1}{2}\left(\Theta^{2}+\left(\Psi_{0}-a_{0}\right)_{\mid 0}-\left(\Phi^{2} \Phi_{4} \Psi^{-2}\right)_{\mid 4}+\Theta_{\alpha}^{\gamma} \Theta_{\gamma}^{\alpha}\right)\right)\right\}, \\
& \text { (f) } \quad \bar{G}_{40}=3 \Phi_{4} \Theta+\left(a_{0}-\Psi_{0}\right) K-\Theta_{\mid 4}+\Psi^{2} a_{\gamma}\left(\Psi^{\gamma}-c^{\gamma}\right) \\
& -\left(\Theta_{\alpha}^{\gamma}+\Phi^{2} \omega_{\alpha}^{\gamma}\right)\left(K_{\gamma}^{\alpha}-\Psi^{2} \eta_{\gamma}^{\alpha}\right)+\Phi^{2}\left\{d_{\gamma}\left(\Phi^{\gamma}-b^{\gamma}\right)\right. \\
& \left.+\frac{1}{2}\left(\Psi^{2} a^{\gamma} \Phi^{-2}-d^{\gamma}\right)_{\mid \gamma}\right\} .
\end{aligned}
$$

Next, we consider the coordinate-free form of the Einstein field equations expressed as follows

$$
\bar{G}(X, Y)+\lambda \bar{g}(X, Y)=c \bar{T}(X, Y), \quad \forall X, Y \in \Gamma(T \bar{M}),
$$

where $\lambda$ and $c$ are constants and $\bar{T}$ is the energy-momentum tensor field of the $5 D$ universe $(\bar{M}, \bar{g})$. The local components of $\bar{T}$ with respect to the adapted frame field are given by 

(a) $\bar{T}_{\alpha \beta}=\bar{T}\left(\frac{\delta}{\delta x^{\beta}}, \frac{\delta}{\delta x^{\alpha}}\right), \quad$ (b) $\bar{T}_{\alpha 0}=\bar{T}\left(\frac{\delta}{\delta x^{0}}, \frac{\delta}{\delta x^{\alpha}}\right)$,
(c) $\bar{T}_{00}=\bar{T}\left(\frac{\delta}{\delta x^{0}}, \frac{\delta}{\delta x^{0}}\right)$,
(d) $\bar{T}_{\alpha 4}=\bar{T}\left(\frac{\partial}{\partial x^{4}}, \frac{\delta}{\delta x^{\alpha}}\right)$,
(e) $\bar{T}_{44}=\bar{T}\left(\frac{\partial}{\partial x^{4}}, \frac{\partial}{\partial x^{4}}\right)$,
(f) $\bar{T}_{40}=\bar{T}\left(\frac{\delta}{\delta x^{0}}, \frac{\partial}{\partial x^{4}}\right)$.

Then, we take $X=\delta / \delta x^{\beta}$ and $Y=\delta / \delta x^{\alpha}$ into (5.4), and by using (5.3a), (5.5) and (2.6), we deduce that

$$
\begin{array}{r}
G_{\alpha \beta}+\left\{\lambda-\frac{1}{2}\left(\Phi^{2} \omega_{\mu}^{\gamma} \omega_{\gamma}^{\mu}-\Psi^{2} \eta_{\mu}^{\gamma} \eta_{\gamma}^{\mu}+2\left(\left(b^{\gamma}+c^{\gamma}-\Phi^{\gamma}-\Psi^{\gamma}\right)_{\mid \gamma}\right.\right.\right. \\
\left.-\left(\Phi^{\gamma}-b^{\gamma}\right)\left(\Phi_{\gamma}-b_{\gamma}\right)-\left(\Psi^{\gamma}-c^{\gamma}\right)\left(\Psi_{\gamma}-c_{\gamma}\right)\right) \\
+\Phi^{-2}\left(\Theta^{2}-2 \Phi_{0} \Theta+2\left(\Psi_{0}-a_{0}\right)\left(\Theta+\Psi_{0}-a_{0}-\Phi_{0}\right)\right. \\
\left.+\left(\Psi_{0}-a_{0}\right)_{\mid 0}+2 \Theta_{\mid 0}-\left(\Phi^{2} \Phi_{4} \Psi^{-2}\right)_{\mid 4}+\Theta_{\mu}^{\gamma} \Theta_{\gamma}^{\mu}\right) \\
\left.\left.-\Psi^{-2}\left(K^{2}+\left(\Phi_{4}-7 \Psi_{4}\right) K+K_{\mid 4}+K_{\mu}^{\gamma} K_{\gamma}^{\mu}\right)\right)\right\} h_{\alpha \beta} \\
+\frac{1}{2}\left(\left(b_{\alpha}+c_{\alpha}-\Phi_{\alpha}-\Psi_{\alpha}\right)_{\mid \beta}+\left(b_{\beta}+c_{\beta}-\Phi_{\beta}-\Psi_{\beta}\right)_{\mid \alpha}\right) \\
+\left(\Phi^{-2} \Theta_{\alpha \beta}\right)_{\mid 0}-\left(\Psi^{-2} K_{\alpha \beta}\right)_{\mid 4}+\Phi^{-2}\left(\Phi_{0}+\Psi_{0}-a_{0}+\Theta\right) \Theta_{\alpha \beta} \\
+\Psi^{-2}\left(\Psi_{4}-\Phi_{4}-K\right) K_{\alpha \beta}+\left(\Phi_{\alpha}-b_{\alpha}\right)\left(\Phi_{\beta}-b_{\beta}\right) \\
-\left(\Psi_{\alpha}-c_{\alpha}\right)\left(\Psi_{\beta}-c_{\beta}\right)=c \bar{T}_{\alpha \beta},
\end{array}
$$

where we put

$$
G_{\alpha \beta}=R_{\alpha \beta}-\frac{R}{2} h_{\alpha \beta} .
$$

We call (5.6) the spatial Einstein field equations (SEFE) of the $5 D$ universe $(\bar{M}, \bar{g})$. Also, we call $G_{\alpha \beta}$ the spatial Einstein gravitational tensor field.

Similarly, taking pairs of vector fields from the adapted frame field into (5.4), we deduce the last 9(EFE) for $(\bar{M}, \bar{g})$ :

$$
\begin{aligned}
& R_{\alpha 0 \gamma}^{\gamma}-\left(\Psi_{\alpha}-c_{\alpha}\right)_{\mid 0}+\left(\Phi^{2} d_{\alpha} \Psi^{-2}-a_{\alpha}\right)_{\mid 4} \\
& -\left(\Psi_{\alpha}-c_{\alpha}\right)\left(\Psi_{0}-a_{0}\right)+\left(\Theta+\Psi_{0}-a_{0}\right)\left(\Phi_{\alpha}-b_{\alpha}\right) \\
& -\left(\Phi^{\gamma}-b^{\gamma}\right)\left(\Theta_{\alpha \gamma}+\Phi^{2} \omega_{\alpha \gamma}\right)+\frac{1}{2}\left\{\left(K-\Phi_{4}-\Psi_{4}\right)\left(\Phi^{2} d_{\alpha} \Psi^{-2}-a_{\alpha}\right)\right. \\
& \left.-\left(\Phi^{2} d^{\gamma} \Psi^{-2}-a^{\gamma}\right)\left(K_{\alpha \gamma}-\Psi^{2} \eta_{\alpha \gamma}\right)\right\}=c \bar{T}_{\alpha 0}, \\
& R_{\alpha 4 \gamma}^{\gamma}-\left(\Phi_{\alpha}-b_{\alpha}\right)_{\mid 4}+\frac{1}{2}\left(\Psi^{2} a_{\alpha} \Phi^{-2}-d_{\alpha}\right)_{\mid 0} \\
& +\left(K+\Phi_{4}\right)\left(\Psi_{\alpha}-c_{\alpha}\right)-\Phi_{4}\left(\Phi_{\alpha}-b_{\alpha}\right)-\left(\Psi^{\gamma}-c^{\gamma}\right)\left(K_{\alpha \gamma}-\Psi^{2} \eta_{\alpha \gamma}\right) \\
& +\frac{1}{2}\left\{\left(\Theta+a_{0}-\Phi_{0}\right) \Psi^{2} a_{\alpha} \Phi^{-2}+\left(a_{0}-\Phi_{0}-\Theta\right) d_{\alpha}\right. \\
& \left.-\left(\Psi^{2} a^{\gamma} \Phi^{-2}-d^{\gamma}\right)\left(\Theta_{\alpha \gamma}+\Phi^{2} \omega_{\alpha \gamma}\right)\right\}=c \bar{T}_{\alpha 4}, \\
& \Theta^{2}+\left(a_{0}-\Psi_{0}\right)_{\mid 0}+\left(\Phi^{2} \Phi_{4} \Psi^{-2}\right)_{\mid 4}+2\left(\Phi_{0}-a_{0}\right) \Theta \\
& +\Phi^{2}\left\{2 \lambda+R+\Phi^{2}\left(d^{\gamma} d_{\gamma} \Psi^{-2}-\omega_{\alpha}^{\gamma} \omega_{\gamma}^{\alpha}\right)-\Psi^{2}\left(a^{\gamma} a_{\gamma} \Phi^{-2}+\eta_{\alpha}^{\gamma} \eta_{\gamma}^{\alpha}\right)\right. \\
& -2\left(\Psi_{\gamma}-c_{\gamma}\right)\left(\Phi^{\gamma}+\Psi^{\gamma}-b^{\gamma}-c^{\gamma}\right)-2 K \Phi_{4} \Psi^{-2}+2 c_{\mid \gamma}^{\gamma}-2 \Psi_{\mid \gamma}^{\gamma} \\
& \left.-\Psi^{-2}\left(K^{2}+\left(\Phi_{4}-7 \Psi_{4}\right) K+K_{\mid 4}+K_{\alpha}^{\gamma} K_{\gamma}^{\alpha}\right)\right\}=2 c \bar{T}_{00},
\end{aligned}
$$




$$
\begin{aligned}
K^{2}+ & \left(\Phi_{4}-\Psi_{4}\right) K-K_{\mid 4}-K_{\alpha}^{\gamma} K_{\gamma}^{\alpha}+\Phi^{2} d_{\gamma} d^{\gamma} \\
+ & \Psi^{2}\left\{2\left(\Phi^{\gamma}-\Psi^{\gamma}+c^{\gamma}-b^{\gamma}\right)\left(\Phi_{\gamma}-b_{\gamma}\right)-2 \lambda-R-\Phi^{2} \omega_{\alpha}^{\gamma} \omega_{\gamma}^{\alpha}\right. \\
- & \Psi^{2} \eta_{\alpha}^{\gamma} \eta_{\gamma}^{\alpha}-\Psi^{2} a_{\gamma} a^{\gamma} \Phi^{-2}+2 \Phi_{\mid \gamma}^{\gamma}-2 b_{\mid \gamma}^{\gamma}-\Psi^{-2}\left(2 \Theta_{\mid 0}-2 \Phi_{0} \Theta\right. \\
+\Theta^{2}+ & \left.\left.\left(\Psi_{0}-a_{0}\right)_{\mid 0}-\left(\Phi^{2} \Phi_{4} \Psi^{-2}\right)_{\mid 4}+\Theta_{\alpha}^{\gamma} \Theta_{\gamma}^{\alpha}\right)\right\}=2 c \bar{T}_{44}, \\
& 3 \Phi_{4} \Theta-\left(\Psi_{0}-a_{0}\right) K-\Theta_{\mid 4}+\Psi^{2} a_{\gamma}\left(\Psi^{\gamma}-c^{\gamma}\right) \\
& -\left(\Theta_{\alpha}^{\gamma}+\Phi^{2} \omega_{\alpha}^{\gamma}\right)\left(K_{\gamma}^{\alpha}-\Psi^{2} \eta_{\gamma}^{\alpha}\right)+\Phi^{2}\left\{d_{\gamma}\left(\Phi^{\gamma}-b^{\gamma}\right)\right. \\
& \left.+\frac{1}{2}\left(\Psi^{2} a^{\gamma} \Phi^{-2}-d^{\gamma}\right)_{\mid \gamma}\right\}=c \bar{T}_{40} .
\end{aligned}
$$

It is worth mentioning that all the (EFE) obtained above are expressed in terms of spatial tensor fields and their covariant derivatives with respect to the Riemannian spatial connection.

\section{Splitting of (EFE) for a $5 D$ Robertson-Walker universe}

In this section we consider the $5 D$ Robertson-Walker universe $(\bar{M}, \bar{g})$ whose Lorentz metric $\bar{g}$ is given by the line element (cf. [2])

$$
d \bar{s}^{2}=-\left(d x^{0}\right)^{2}+f^{2}\left(x^{0}, x^{4}\right) g_{\alpha \beta}\left(x^{1}, x^{2}, x^{3}\right) d x^{\alpha} d x^{\beta}+\left(d x^{4}\right)^{2} .
$$

In this case we have

$$
\begin{gathered}
\Phi_{a}=\Psi_{a}=0, \quad \frac{\delta}{\delta x^{i}}=\frac{\partial}{\partial x^{i}}, \quad a_{i}=A_{i}=0, \\
b_{\alpha}=c_{\alpha}=d_{\alpha}=B_{\alpha}=0, \omega_{\alpha \beta}=\eta_{\alpha \beta}=0,
\end{gathered}
$$

for all $a \in\{0,1,2,3,4\}, i \in\{0,1,2,3\}, \alpha, \beta, \ldots \in\{1,2,3\}$. As the spatial Riemannian metric $h$ is given by

$$
h_{\alpha \beta}=f^{2} g_{\alpha \beta}, \quad h^{\alpha \beta}=f^{-2} g_{\alpha \beta},
$$

from (2.9) and (2.10) we deduce that

$$
\begin{aligned}
& \Theta_{\alpha \beta}=f \frac{\partial f}{\partial x^{0}} g_{\alpha \beta}, \quad \Theta_{\alpha}^{\gamma}=f^{-1} \frac{\partial f}{\partial x^{0}} \delta_{\alpha}^{\gamma}, \\
& K_{\alpha \beta}=f \frac{\partial f}{\partial x^{4}} g_{\alpha \beta}, \quad K_{\alpha}^{\gamma}=f^{-1} \frac{\partial f}{\partial x^{4}} \delta_{\alpha}^{\gamma},
\end{aligned}
$$

and

$$
\Theta=3 f^{-1} \frac{\partial f}{\partial x^{0}}, \quad K=3 f^{-1} \frac{\partial f}{\partial x^{4}},
$$

respectively. Also, from (2.11) we obtain

$$
\sigma_{\alpha \beta}=H_{\alpha \beta}=0 .
$$

Next, we note that the spatial distribution of a $5 D$ Robertson-Walker universe is integrable, and its leaves are locally given by $x^{0}=$ constant, $x^{4}=$ constant. Thus from (6.4) we deduce that

$$
\Theta_{\alpha \beta}=K_{\alpha \beta}=0,
$$

on the leaves of $\mathcal{S} \bar{M}$. Then, by using (6.2), (6.6) and (6.7) we can state the following result.

Theorem 6.1. Let $(\bar{M}, \bar{g})$ be a $5 D$ Robertson-Walker universe. Then we have the following assertions:

(i) $(\bar{M}, \bar{g})$ is both vorticity-free and shear-free $5 D$ universe.

(ii) The leaves of the spatial distribution are totally geodesic immersed in $(\bar{M}, \bar{g})$.

Now, from (2.15)we see that the local coefficients of the Riemannian spatial connection on $(\bar{M}, \bar{g})$ are given by 


$$
\begin{aligned}
& \text { (a) } \Gamma_{\alpha \beta}^{\gamma}=\frac{1}{2} g^{\gamma \mu}\left\{\frac{\partial g_{\mu \alpha}}{\partial x^{\beta}}+\frac{\partial g_{\mu \beta}}{\partial x^{\alpha}}-\frac{\partial g_{\alpha \beta}}{\partial x^{\mu}}\right\}, \\
& \text { (b) } \Gamma_{\alpha 0}^{\gamma}=f^{-1} \frac{\partial f}{\partial x^{0}} \delta_{\alpha}^{\gamma}, \quad \text { (c) } \Gamma_{\alpha 4}^{\gamma}=f^{-1} \frac{\partial f}{\partial x^{4}} \delta_{\alpha}^{\gamma} .
\end{aligned}
$$

By using (6.8a) into (3.3a) we obtain

$$
R_{\alpha \beta \gamma}^{\mu}=\frac{\partial \Gamma_{\alpha \beta}^{\mu}}{\partial x^{\gamma}}-\frac{\partial \Gamma_{\alpha \gamma}^{\mu}}{\partial x^{\beta}}+\Gamma_{\alpha \beta}^{\nu} \Gamma_{\nu \gamma}^{\mu}-\Gamma_{\alpha \gamma}^{\nu} \Gamma_{\nu \beta}^{\mu} .
$$

Thus $R_{\alpha \beta}$ from (4.7) depend only on variables $\left(x^{1}, x^{2}, x^{3}\right)$ and represent the local coefficients of a Ricci tensor of a Riemannian manifold with the metric $g_{\alpha \beta}\left(x^{1}, x^{2}, x^{3}\right)$. However, by (4.10) the spatial scalar curvature is given by

$$
R=f^{-2} g^{\gamma \mu} R_{\gamma \mu},
$$

and therefore, in general, it depends on all variables $\left(x^{a}\right)$. Also, by using (6.8) and (6.2) into (3.3b), (3.3c) and (3.3d), we deduce that

$$
\text { (a) } R_{\alpha 0 \gamma}^{\mu}=0, \quad(b) \quad R_{\alpha 4 \gamma}^{\mu}=0, \quad(c) \quad R_{\alpha 40}^{\mu}=0 .
$$

According to (6.9) and (6.10) we can state the following theorem.

Theorem 6.2. (i) The local components of the curvature tensor field of the Riemannian spatial connection $\nabla$ depend only on $\left(x^{1}, x^{2}, x^{3}\right)$.

(ii) The curvature of $\nabla$ coincides with the curvature of a Riemannian manifold with metric $g_{\alpha \beta}\left(x^{1}, x^{2}, x^{3}\right)$.

Next, from (6.4) and (6.5) we obtain the following covariant derivatives:

$$
\begin{aligned}
& \Theta_{\alpha \beta \mid 0}=\left\{f \frac{\partial^{2} f}{\left(\partial x^{0}\right)^{2}}-\left(\frac{\partial f}{\partial x^{0}}\right)^{2}\right\} g_{\alpha \beta}, \\
& K_{\alpha \beta \mid 4}=\left\{f \frac{\partial^{2} f}{\left(\partial x^{4}\right)^{2}}-\left(\frac{\partial f}{\partial x^{4}}\right)^{2}\right\} g_{\alpha \beta}, \\
& \Theta_{\mid 0}=3 f^{-2}\left\{f \frac{\partial^{2} f}{\left(\partial x^{0}\right)^{2}}-\left(\frac{\partial f}{\partial x^{0}}\right)^{2}\right\}, \\
& K_{\mid 4}=3 f^{-2}\left\{f \frac{\partial^{2} f}{\left(\partial x^{4}\right)^{2}}-\left(\frac{\partial f}{\partial x^{4}}\right)^{2}\right\} .
\end{aligned}
$$

Then, by direct calculations using (6.2), (6.4), (6.5), (6.10) and (6.11) into (5.6) we obtain the following six spatial Einstein field equations for the $5 D$ Robertson-Walker universe:

$$
\begin{aligned}
G_{\alpha \beta}+\left\{\lambda f^{2}+\frac{1}{2}\left(f \frac{\partial^{2} f}{\left(\partial x^{4}\right)^{2}}\right.\right. & \left.+5\left(\frac{\partial f}{\partial x^{4}}\right)^{2}\right)-2 f \frac{\partial^{2} f}{\left(\partial x^{0}\right)^{2}} \\
& \left.-\left(\frac{\partial f}{\partial x^{0}}\right)^{2}\right\} g_{\alpha \beta}=c \bar{T}_{\alpha \beta} .
\end{aligned}
$$

In a similar way, from (5.8)-(5.12) we deduce that the last nine (EFE) for $(\bar{M}, \bar{g})$ are given by

$$
\begin{gathered}
(a) \bar{T}_{\alpha 0}=0, \quad \text { (b) } \bar{T}_{\alpha 4}=0 \\
9 f^{-2}\left(\left(\frac{\partial f}{\partial x^{0}}\right)^{2}-\left(\frac{\partial f}{\partial x^{4}}\right)^{2}\right)-3 f^{-1} \frac{\partial^{2} f}{\left(\partial x^{4}\right)^{2}}+2 \lambda+R=2 c \bar{T}_{00} \\
3 f^{-2}\left\{3\left(\frac{\partial f}{\partial x^{4}}\right)^{2}-2\left(\frac{\partial f}{\partial x^{0}}\right)^{2}-f \frac{\partial^{2} f}{\left(\partial x^{4}\right)^{2}}-2 f \frac{\partial^{2} f}{\left(\partial x^{0}\right)^{2}}\right\} \\
-2 \lambda-R=2 c \bar{T}_{44}, \\
3 f^{-1} \frac{\partial^{2} f}{\left(\partial x^{0}\right)^{2}}=-c \bar{T}_{40} .
\end{gathered}
$$


We note that the equations from (6.13) have been obtained from (5.8) and (5.9) taking into account (6.2), (6.10a) and $(6.10 \mathrm{~b})$, and using the hypothesis that $c$ is a positive constant. As a consequence of (6.13) we see that there are only 9 local components of the energy-momentum tensor field which are not yet determined so far. Also, we note that by adding (6.14) and (6.15), we obtain

$$
\begin{array}{r}
3 f^{-2}\left\{\left(\frac{\partial f}{\partial x^{0}}\right)^{2}-2 f \frac{\partial^{2} f}{\left(\partial x^{4}\right)^{2}}-f \frac{\partial^{2} f}{\left(\partial x^{0}\right)^{2}}\right\} \\
=2 c\left(\bar{T}_{00}+\bar{T}_{44}\right) .
\end{array}
$$

Thus the warping function $f\left(x^{0}, x^{4}\right)$ must satisfy two PDE given by (6.16) and (6.17).

\section{Conclusions}

The splitting of (EFE) is performed with respect to the $(1+1+3)$ threading of a $5 D$ universe developed by the first author in [1,2]. We obtain six (SEFE) given by (5.6) and other nine (EFE) given by [(5.8)-(5.12)]. It is worth mentioning that such a splitting was expressed in terms of spatial tensor fields and by using the Riemannian spatial connection. This approach is totally different from the ones performed in both the brane-world theory $[3,4]$ and the space-time-matter theory $[5,6]$. The main differences are consequences of the geometrical configuration of a $5 D$ universe we consider in the paper. Namely, in our approach the $4 D$ spacetime $M$ is the base of a submersion on the $5 D$ universe of $\bar{M}$, while in the above mentioned references, $M$ is an embedded submanifold of $\bar{M}$.

In particular, we obtain the splitting of (EFE) in a $5 D$ Robertson-Walker universe given by [(6.12)-(6.16)]. The simplicity of these equations is stressing the main role of spatial tensor fields into the study. The vanishing of the six local components of the energy-momentum tensor field $\bar{T}$ [cf.(6.13)], and the two PDE satisfied by the warping function [cf.(6.16), (6.17)] are important constraints on the geometrical configuration of a $5 D$ Robertson-Walker universe. For example, if $\bar{T}_{40}=0$, then from (6.16) we conclude that the warping function must be linear function with respect to the time coordinate.

Finally, we should mention that our study is mainly developed on the mathematical part of Einstein field equations in a $5 D$ universe $\bar{M}$. The experimental physics might bring more light on the local components of the energy-momentum tensor field of $\bar{M}$. This will bring more insights on the $4 D$ physics under the assumption of the existence of the fifth dimension.

\section{Appendix}

In order to obtain the formulas for the local components of $\bar{R}$ from Sect. 3, we start with the well known formula

$$
\bar{R}(X, Y, Z)=\bar{\nabla}_{X} \bar{\nabla}_{Y} Z-\bar{\nabla}_{Y} \bar{\nabla}_{X} Z-\bar{\nabla}_{[X, Y]} Z,
$$

for all $X, Y, Z \in \Gamma(T \bar{M})$. Then by using (2.16a), (2.16d) and (2.16e), we obtain

$$
\begin{aligned}
& \bar{\nabla}_{\frac{\delta}{\delta x \gamma}} \bar{\nabla}_{\frac{\delta}{\delta x^{\beta}}} \frac{\delta}{\delta x^{\alpha}}=\left\{\frac{\delta \Gamma_{\alpha \beta}^{\delta}}{\delta x^{\gamma}}+\Gamma_{\alpha \beta}^{\mu} \Gamma_{\mu \gamma}^{\delta}+\left(\omega_{\alpha \beta}+\Phi^{-2} \Theta_{\alpha \beta}\right) \Gamma_{\gamma 0}^{\delta}\right. \\
& \left.+\left(\eta_{\alpha \beta}-\Psi^{-2} K_{\alpha \beta}\right) \Gamma_{\gamma 4}^{\delta}\right\} \frac{\delta}{\delta x^{\delta}}+\left\{\left(\omega_{\mu \gamma}+\Phi^{-2} \Theta_{\mu \gamma}\right) \Gamma_{\alpha \beta}^{\mu}+\frac{\delta \omega_{\alpha \beta}}{\delta x^{\gamma}}\right. \\
& -2 \Phi^{-2} \Phi_{\gamma} \Theta_{\alpha \beta}+\Phi^{-2} \frac{\delta \Theta_{\alpha \beta}}{\delta x^{\gamma}}+\frac{1}{2}\left(\Psi^{2} a_{\gamma} \Phi^{-2}+d_{\gamma}\right)\left(\eta_{\alpha \beta}-\Psi^{-2} K_{\alpha \beta}\right) \\
& \left.+\left(\omega_{\alpha \beta}+\Phi^{-2} \Theta_{\alpha \beta}\right) \Phi_{\gamma}\right\} \frac{\delta}{\delta x^{0}}+\left\{\frac{\delta \eta_{\alpha \beta}}{\delta x^{\gamma}}+2 \Psi^{-2} \Psi_{\gamma} K_{\alpha \beta}-\Psi^{-2} \frac{\delta K_{\alpha \beta}}{\delta x^{\gamma}}\right. \\
& \left.+\left(\eta_{\alpha \beta}-\Psi^{-2} K_{\alpha \beta}\right) \Psi_{\gamma}+\frac{1}{2}\left(\Phi^{2} d_{\gamma} \Psi^{-2}+a_{\gamma}\right)\left(\omega_{\alpha \beta}+\Phi^{-2} \Theta_{\alpha \beta}\right)\right\} \frac{\partial}{\partial x^{4}} .
\end{aligned}
$$


Also, by using (2.12a), (2.16b) and (2.16c), we deduce that

$$
\begin{aligned}
& \bar{\nabla}_{\left[\frac{\delta}{\delta x^{\gamma}}, \frac{\delta}{\delta x^{\beta}}\right]} \frac{\delta}{\delta x^{\alpha}}=2\left\{\omega_{\beta \gamma} \Gamma_{\alpha 0}^{\delta}+\eta_{\beta \gamma} \Gamma_{\alpha}^{\delta}\right\} \frac{\delta}{\delta x^{\delta}} \\
& +2\left\{\left(\Phi_{\alpha}-b_{\alpha}\right) \omega_{\beta \gamma}+\frac{1}{2}\left(\Psi^{2} a_{\alpha} \Phi^{-2}-d_{\alpha}\right) \eta_{\beta \gamma}\right\} \frac{\delta}{\delta x^{0}} \\
& +2\left\{\left(\Psi_{\alpha}-c_{\alpha}\right) \eta_{\beta \gamma}+\frac{1}{2}\left(\Phi^{2} d_{\alpha} \Psi^{-2}-a_{\alpha}\right) \omega_{\beta \gamma}\right\} \frac{\partial}{\partial x^{4}} .
\end{aligned}
$$

Next, by using $\left(A_{1}\right),\left(A_{2}\right),\left(A_{3}\right)$, and taking into account (3.3a), we obtain

$$
\begin{array}{r}
\bar{R}\left(\frac{\delta}{\delta x^{\gamma}}, \frac{\delta}{\delta x^{\beta}}, \frac{\delta}{\delta x^{\alpha}}\right)=\left\{R_{\alpha \beta \gamma}^{\mu}+\mathcal{A}_{(\beta \gamma)}\left(\left(\omega_{\alpha \beta}\right.\right.\right. \\
\left.\left.\left.+\Phi^{-2} \Theta_{\alpha \beta}\right) \Gamma_{\gamma 0}^{\mu}+\left(\eta_{\alpha \beta}-\Psi^{-2} K_{\alpha \beta}\right) \Gamma_{\gamma 4}^{\mu}\right)\right\} \frac{\delta}{\delta x^{\mu}} \\
+\left\{\mathcal { A } _ { ( \beta \gamma ) } \left(\omega_{\alpha \beta \mid \gamma}+\Phi^{-2}\left(\Theta_{\alpha \beta \mid \gamma}+\Theta_{\alpha \gamma} \Phi_{\beta}\right)+\omega_{\alpha \beta} \Phi_{\gamma}\right.\right. \\
\left.+\frac{1}{2}\left(\Psi^{2} a_{\gamma} \Phi^{-2}+d_{\gamma}\right)\left(\eta_{\alpha \beta}-\Psi^{-2} K_{\alpha \beta}\right)\right) \\
\left.-2\left(\Phi_{\alpha}-b_{\alpha}\right) \omega_{\beta \gamma}-\left(\Psi^{2} a_{\alpha} \Phi^{-2}-d_{\alpha}\right) \eta_{\beta \gamma}\right\} \frac{\delta}{\delta x^{0}} \\
+\left\{\mathcal { A } _ { ( \beta \gamma ) } \left(\eta_{\alpha \beta \mid \gamma}+\Psi^{-2}\left(K_{\alpha \gamma \mid \beta}+K_{\alpha \beta} \Psi_{\gamma}\right)\right.\right. \\
\left.+\eta_{\alpha \beta} \Psi_{\gamma}+\frac{1}{2}\left(\Phi^{2} d_{\gamma} \Psi^{-2}+a_{\gamma}\right)\left(\omega_{\alpha \beta}+\Psi^{-2} \Theta_{\alpha \beta}\right)\right) \\
\left.-2\left(\Psi_{\alpha}-c_{\alpha}\right) \eta_{\beta \gamma}-\left(\Phi^{2} d_{\alpha} \Psi^{-2}-a_{\alpha}\right) \omega_{\beta \gamma}\right\} \frac{\partial}{\partial x^{4}} .
\end{array}
$$

By similar calculations using $\left(A_{1}\right),(2.12),(2.14),(3.3)$ and the covariant derivatives induced by the Riemannian spatial connection, we deduce that

$$
\begin{array}{r}
\bar{R}\left(\frac{\delta}{\delta x^{\gamma}}, \frac{\delta}{\delta x^{0}}, \frac{\delta}{\delta x^{\alpha}}\right)=\left\{R_{\alpha 0 \gamma}^{\mu}+\left(\Phi_{\alpha}-b_{\alpha}\right) \Gamma_{\gamma 0}^{\mu}\right. \\
+\frac{1}{2}\left(\Phi^{2} d_{\alpha} \Psi^{-2}-a_{\alpha}\right) \Gamma_{\gamma 4}^{\mu}-\left(\Theta_{\alpha \gamma}+\Phi^{-2} \omega_{\alpha \gamma}\right)\left(\Phi^{\mu}-b^{\mu}\right) \\
\left.-\frac{1}{2}\left(\eta_{\alpha \gamma}-\Psi^{-2} K_{\alpha \gamma}\right)\left(\Psi^{2} a^{\mu}-\Phi^{2} d^{\mu}\right)\right\} \frac{\delta}{\delta x^{\mu}} \\
+\left\{\Phi_{\alpha \mid \gamma}-b_{\alpha \mid \gamma}-\left(\omega_{\alpha \gamma}+\Phi^{-2} \Theta_{\alpha \gamma}\right) \mid 0\right. \\
-\left(\omega_{\alpha \mu}+\Phi^{-2} \Theta_{\alpha \mu}\right) \Gamma_{\gamma 0}^{\mu}+\left(\Phi_{\alpha}-b_{\alpha}\right)\left(\Phi_{\gamma}-b_{\gamma}\right) \\
-\Phi_{0}\left(\omega_{\alpha \gamma}+\Phi^{-2} \Theta_{\alpha \gamma}\right)-\Phi_{4}\left(\eta_{\alpha \gamma}-\Psi^{-2} K_{\alpha \gamma}\right) \\
\left.+\frac{1}{4}\left(3 d_{\alpha} a_{\gamma}-a_{\alpha} d_{\gamma}+\Phi^{2} d_{\alpha} d_{\gamma} \Psi^{-2}-3 \Psi^{2} a_{\alpha} a_{\gamma} \Phi^{-2}\right)\right\} \frac{\delta}{\delta x^{0}} \\
\left\{\frac{1}{2}\left(\Phi^{2} d_{\alpha} \Psi^{-2}-a_{\alpha}\right)\left|\gamma-\left(\eta_{\alpha \gamma}-\Psi^{-2} K_{\alpha \gamma}\right)\right| 0\right. \\
-\left(\eta_{\alpha \mu}-\Psi^{-2} K_{\alpha \mu}\right) \Gamma_{\gamma 0}^{\mu}+\frac{1}{2}\left(\Phi_{\alpha}-b_{\alpha}\right)\left(\Phi^{2} d_{\gamma} \Psi^{-2}+a_{\gamma}\right) \\
+\frac{1}{2}\left(\Psi_{\gamma}-c_{\gamma}\right)\left(\Phi^{2} d_{\alpha} \Psi^{-2}-a_{\alpha}\right) \\
-\Phi^{2} \Phi_{4} \Psi^{-2}\left(\omega_{\alpha \gamma}+\Phi^{-2} \Theta_{\alpha \gamma}\right) \\
\left.-\Psi_{0}\left(\eta_{\alpha \gamma}-\Psi^{-2} K_{\alpha \gamma}\right)-\left(\Psi_{\gamma}-c_{\gamma}\right) a_{\gamma}\right\} \frac{\partial}{\partial x^{4}},
\end{array}
$$




$$
\begin{array}{r}
\bar{R}\left(\frac{\delta}{\delta x^{\gamma}}, \frac{\partial}{\partial x^{4}}, \frac{\delta}{\delta x^{\alpha}}\right)=\left\{R_{\alpha 4 \gamma}^{\mu}+\frac{1}{2}\left(\Psi^{2} a_{\alpha} \Phi^{-2}-d_{\alpha}\right) \Gamma_{\gamma 0}^{\mu}\right. \\
+\left(\Psi_{\alpha}-c_{\alpha}\right) \Gamma_{\gamma 4}^{\mu}-\frac{1}{2}\left(\omega_{\alpha \gamma}+\Phi^{-2} \Theta_{\alpha \gamma}\right)\left(\Psi^{2} a^{\mu}-\Phi^{2} d^{\mu}\right) \\
\left.+\Psi^{2}\left(\eta_{\alpha \gamma}-\Psi^{-2} K_{\alpha \gamma}\right)\left(c^{\mu}-\Psi^{\mu}\right)\right\} \frac{\delta}{\delta x^{\mu}}+\left\{\frac{1}{2}\left(\Psi^{2} a_{\alpha} \Phi^{-2}-d_{\alpha}\right)_{\mid \gamma}\right. \\
-\left(\omega_{\alpha \gamma}+\Phi^{2} \Theta_{\alpha \gamma}\right)_{\mid 4}-\left(\omega_{\alpha \mu}+\Phi^{-2} \Theta_{\alpha \mu}\right) \Gamma_{\gamma}^{\mu}+\frac{1}{2}\left(\Psi^{2} a_{\alpha} \Phi^{-2}-d_{\alpha}\right) \Psi_{\gamma} \\
+\frac{1}{2}\left(\Psi_{\alpha}-c_{\alpha}\right)\left(\Psi^{2} a_{\gamma} \Phi^{-2}+d_{\gamma}\right)-\Phi_{4}\left(\omega_{\alpha \gamma}+\Phi^{-2} \Theta_{\alpha \gamma}\right)-\left(\Phi_{\alpha}-b_{\alpha}\right) d_{\gamma} \\
\left.-\Psi^{2} \Phi^{-2}\left(\Psi_{0}-a_{0}\right)\left(\eta_{\alpha \gamma}-\Psi^{-2} K_{\alpha \gamma}\right)-\frac{1}{2}\left(\Psi^{2} a_{\alpha} \Phi^{-2}-d_{\alpha}\right) c_{\gamma}\right\} \frac{\delta}{\delta x^{0}} \\
+\left\{\left(\Psi_{\alpha}-c_{\alpha}\right)_{\mid \gamma}-\left(\eta_{\alpha \gamma}-\Psi^{-2} K_{\alpha \gamma}\right)_{\mid 4}-\left(\eta_{\alpha \mu}-\Psi^{-2} K_{\alpha \mu}\right) \Gamma_{\gamma 4}^{\mu}\right. \\
+\frac{1}{4}\left(a_{\alpha}-\Phi^{2} d_{\alpha} \Psi^{-2}\right)\left(3 d_{\gamma}+\Psi^{2} a_{\gamma} \Phi^{-2}\right) \\
+\left(\Psi_{\alpha}-c_{\alpha}\right)\left(\Psi_{\gamma}-c_{\gamma}\right)+\Psi_{4}\left(\eta_{\alpha \gamma}-\Psi^{-2} K_{\alpha \gamma}\right) \\
\left.-\left(\Psi_{0}-a_{0}\right)\left(\omega_{\alpha \gamma}+\Phi^{-2} \Theta_{\alpha \gamma}\right)\right\} \frac{\partial}{\partial x^{4}} . \\
\bar{R}\left(\frac{\delta}{\delta x^{0}}, \frac{\partial}{\partial x^{4}}, \frac{\delta}{\delta x^{\alpha}}\right)=\left\{R_{\alpha 40}^{\mu}+\frac{1}{2}\left(( \Psi ^ { 2 } a _ { \alpha } - \Phi ^ { 2 } d _ { \alpha } ) \left(\Phi^{\mu}-\Psi^{\mu}\right.\right.\right. \\
\left.\left.\left.+c^{\mu}-b^{\mu}\right)+\left(\Psi_{\alpha}-\Phi_{\alpha}+b_{\alpha}-c_{\alpha}\right)\left(\Psi^{2} a^{\mu}-\Phi^{2} d^{\mu}\right)\right)\right\} \frac{\delta}{\delta x^{\mu}} \\
+\left\{\frac{1}{2}\left(\Psi^{2} a_{\alpha} \Phi^{-2}-d_{\alpha}\right)_{\mid 0}+\left(\Phi_{\alpha}-b_{\alpha}\right)_{\mid 4}\right. \\
\left.+\Phi_{4}\left(\Psi_{\alpha}-\Phi_{\alpha}+b_{\alpha}-c_{\alpha}\right)+\frac{1}{2}\left(\Phi_{0}-\Psi_{0}\right)\left(\Psi^{2} a_{\alpha} \Phi^{-2}-d_{\alpha}\right)\right\} \frac{\delta}{\delta x^{0}} \\
+\left\{\left(\Psi_{\alpha}-c_{\alpha}\right)_{\mid 0}-\frac{1}{2}\left(\Phi^{2} d_{\alpha} \Psi^{-2}-a_{\alpha}\right)_{\mid 4}\right. \\
+\left(\Psi_{0}-a_{0}\right)\left(\Psi_{\alpha}-\Phi_{\alpha}+b_{\alpha}-c_{\alpha}\right) \\
\left.-\frac{1}{2}\left(\Phi_{4}+\Psi_{4}\right)\left(\Phi^{2} d_{\alpha} \psi^{-2}-a_{\alpha}\right)\right\} \frac{\partial}{\partial x^{4}} .
\end{array}
$$

Finally we consider the curvature tensor fields $\bar{R}$ and $R$ of the type $(0,4)$ of $\bar{\nabla}$ and $\nabla$ respectively, given by

$$
\begin{aligned}
& \text { (a) } \bar{R}(X, Y, Z, U)=\bar{g}(\bar{R}(X, Y, Z), U), \\
& \text { (b) } R(X, Y, \mathcal{S} Z, \mathcal{S} U)=h(R(X, Y, \mathcal{S} U), \mathcal{S} Z),
\end{aligned}
$$

for all $X, Y, Z, U \in \Gamma(T \bar{M})$. The local components of these tensor fields are listed below:

(a) $\bar{R}_{\alpha \beta \gamma \nu}=\bar{R}\left(\frac{\delta}{\delta x^{\nu}}, \frac{\delta}{\delta x^{\gamma}}, \frac{\delta}{\delta x^{\beta}}, \frac{\delta}{\delta x^{\alpha}}\right)=h_{\beta \mu} \bar{R}_{\alpha \gamma \nu}^{\mu}$,

(b) $\bar{R}_{\alpha 0 \gamma \nu}=\bar{R}\left(\frac{\delta}{\delta x^{\nu}}, \frac{\delta}{\delta x^{\gamma}}, \frac{\delta}{\delta x^{0}}, \frac{\delta}{\delta x^{\alpha}}\right)=-\Phi^{2} \bar{R}_{\alpha \gamma \nu}^{0}$

(c) $\bar{R}_{\alpha \beta 0 \nu}=\bar{R}\left(\frac{\delta}{\delta x^{\nu}}, \frac{\delta}{\delta x^{0}}, \frac{\delta}{\delta x^{\beta}}, \frac{\delta}{\delta x^{\alpha}}\right)=h_{\beta \mu} \bar{R}_{\alpha 0 \nu}^{\mu}$,

(d) $\bar{R}_{\alpha 00 \nu}=\bar{R}\left(\frac{\delta}{\delta x^{\nu}}, \frac{\delta}{\delta x^{0}}, \frac{\delta}{\delta x^{0}}, \frac{\delta}{\delta x^{\alpha}}\right)=-\Phi^{2} \bar{R}_{\alpha}^{0} 0 \nu$,

(e) $\bar{R}_{\alpha 4 \gamma \nu}=\bar{R}\left(\frac{\delta}{\delta x^{\nu}}, \frac{\delta}{\delta x^{\gamma}}, \frac{\partial}{\partial x^{4}}, \frac{\delta}{\delta x^{\alpha}}\right)=\Psi^{2} \bar{R}_{\alpha \gamma \nu}^{4}$,

(f) $\bar{R}_{\alpha \beta 4 \nu}=\bar{R}\left(\frac{\delta}{\delta x^{\nu}}, \frac{\partial}{\partial x^{4}}, \frac{\delta}{\delta x^{\beta}}, \frac{\delta}{\delta x^{\alpha}}\right)=h_{\beta \mu} \bar{R}_{\alpha 4 \nu}^{\mu}$,

(g) $\bar{R}_{\alpha 44 \nu}=\bar{R}\left(\frac{\delta}{\delta x^{\nu}}, \frac{\partial}{\partial x^{4}}, \frac{\partial}{\partial x^{4}}, \frac{\delta}{\delta x^{\alpha}}\right)=\Psi^{2} \bar{R}_{\alpha 4 \nu}^{4}$,

(h) $\bar{R}_{\alpha 04 \nu}=\bar{R}\left(\frac{\delta}{\delta x^{\nu}}, \frac{\partial}{\partial x^{4}}, \frac{\delta}{\delta x^{0}}, \frac{\delta}{\delta x^{\alpha}}\right)=-\Phi^{2} \bar{R}_{\alpha 4 \nu}^{0}$,

(i) $\bar{R}_{\alpha 40 \nu}=\bar{R}\left(\frac{\delta}{\delta x^{\nu}}, \frac{\delta}{\delta x^{0}}, \frac{\partial}{\partial x^{4}}, \frac{\delta}{\delta x^{\alpha}}\right)=\Psi^{2} \bar{R}_{\alpha 0 \nu}^{4}$,

(j) $\bar{R}_{\alpha 040}=\bar{R}\left(\frac{\delta}{\delta x^{0}}, \frac{\partial}{\partial x^{4}}, \frac{\delta}{\delta x^{0}}, \frac{\delta}{\delta x^{\alpha}}\right)=-\Phi^{2} \bar{R}_{\alpha 40}^{0}$,

(k) $\bar{R}_{\alpha 440}=\bar{R}\left(\frac{\delta}{\delta x^{0}}, \frac{\partial}{\partial x^{4}}, \frac{\partial}{\partial x^{4}}, \frac{\delta}{\delta x^{\alpha}}\right)=\Psi^{2} \bar{R}_{\alpha 40}^{4}$,

(l) $\bar{R}_{0404}=\bar{R}\left(\frac{\partial}{\partial x^{4}}, \frac{\delta}{\delta x^{0}}, \frac{\partial}{\partial x^{4}}, \frac{\delta}{\delta x^{0}}\right)=\Psi^{2} \bar{R}_{004}^{4}$, 
and
(a) $R_{\alpha \beta \gamma \nu}=R\left(\frac{\delta}{\delta x^{\nu}}, \frac{\delta}{\delta x^{\gamma}}, \frac{\delta}{\delta x^{\beta}}, \frac{\delta}{\delta x^{\alpha}}\right)=h_{\beta \mu} R_{\alpha \gamma \nu}^{\mu}$,
(b) $R_{\alpha \beta 0 \nu}=R\left(\frac{\delta}{\delta x^{\nu}}, \frac{\delta}{\delta x^{0}}, \frac{\delta}{\delta x^{\beta}}, \frac{\delta}{\delta x^{\alpha}}\right)=h_{\beta \mu} R_{\alpha 0 \nu}^{\mu}$,
(c) $R_{\alpha \beta 4 \nu}=R\left(\frac{\delta}{\delta x^{\nu}}, \frac{\partial}{\partial x^{4}}, \frac{\delta}{\delta x^{\beta}}, \frac{\delta}{\delta x^{\alpha}}\right)=h_{\beta \mu} R_{\alpha 4 \nu}^{\mu}$,
(f) $R_{\alpha \beta 40}=R\left(\frac{\delta}{\delta x^{0}}, \frac{\partial}{\partial x^{4}}, \frac{\delta}{\delta x^{\beta}}, \frac{\delta}{\delta x^{\alpha}}\right)=h_{\beta \mu} R_{\alpha 40}^{\mu}$.

\section{References}

[1] Bejancu, A.:Kinematic quantities and Raychaudhuri equations in a 5D universe. The European Physical Journal C. 75 (7), 346 (2015).

[2] Bejancu, A.:Equations of motion with respect to the $(1+1+3)$ threading of a 5 D universe. The European Physical Journal C. 76 (1), 2 (2016).

[3] Langlois, D.: Brane cosmology. Progress of Theoretical Physics Supplement. 148, 181-212 (2002).

[4] Maartens, R., Kazuya K.: Brane-world gravity. Living Reviews in Relativity. 13 (1), 5 (2010).

[5] Overduin, J.M., Wesson, P.S.: Kaluza-klein gravity. Physics Reports. 283 (5-6), 303-378 (1997).

[6] Wesson, P.S.: Space-Time-Matter: Modern Kaluza-Klein Theory. World Sicentific. Singapore (1999).

\section{Affiliations}

AUREL BEJANCU

AdDress: Universitatea "Al. I. Cuza" IASI, Seminarul Matematic "Al. Myller", B-dul Carol I, No.11, 700506, IASI, Romania.

ORCID ID: 0000-0002-5819-2557

HANI REDA FARRAN

AdDress: Kuwait University Dept. of Mathematics, P.O. Box 5969, Safat 13060. Kuwait.

E-MAIL: hani.farran@ku.edu.kw

ORCID ID: 0000-0002-1397-7406 